\title{
Fluorescent Supramolecular Polymers Formed by Crown Ether-Based Host-Guest Interaction
}

\section{OPEN ACCESS}

Edited by:

Yong Yao,

Nantong University, China

Reviewed by:

Bingbing Shi,

Northwest Normal University, China

Da-Hui Qu,

East China University of Science and

Technology, China

Chen Lin,

Nanjing Normal University, China

Shengyi Dong,

Hunan University, China

*Correspondence:

Tian $\mathrm{He}$

het@hznu.edu.cn

Yang Li

oceanlee136@163.com

Shouchun Yin

yinsc@hznu.edu.cn

Specialty section:

This article was submitted to

Supramolecular Chemistry,

a section of the journal

Frontiers in Chemistry

Received: 17 April 2020

Accepted: 02 June 2020

Published: 24 July 2020

Citation:

Zhang J, Qiu H, He T, Li Y and Yin S (2020) Fluorescent Supramolecular Polymers Formed by Crown Ether-Based Host-Guest Interaction.

Front. Chem. 8:560.

doi: $10.3389 /$ fchem.2020.00560
Jinjin Zhang ${ }^{1}$, Huayu Qiu ${ }^{1,2}$, Tian $\mathrm{He}^{1 *}$, Yang $\mathrm{Li}^{1 *}$ and Shouchun Yin ${ }^{1 *}$

${ }^{1}$ College of Material, Chemistry and Chemical Engineering, Hangzhou Normal University, Hangzhou, China, ${ }^{2}$ Key Laboratory of Organosilicon Chemistry and Materials Technology of Ministry of Education, Hangzhou Normal University, Hangzhou, China

Inspired by the vast array of assemblies present in nature, supramolecular chemistry has attracted significant attention on account of its diverse supra-structures, which include micelles, vesicles, and fibers, in addition to its extensive applications in luminescent materials, sensors, bioimaging, and drug delivery over the past decades. Supramolecular polymers, which represent a combination of supramolecular chemistry and polymer science, are constructed by non-covalent interactions, such as host-guest interactions, hydrogen bonding, hydrophobic or hydrophilic interactions, metal-ligand interactions, $\pi-\pi$ stacking, and electrostatic interactions. To date, numerous host-guest recognition systems have been reported, including crown ethers, cyclodextrins, calixarenes, cucurbituril, pillararenes, and other macrocyclic hosts. Among them, crown ethers, as the first generation of macrocyclic hosts, provide a promising and facile alternative route to supramolecular polymers. In addition, the incorporation of fluorophores into supramolecular polymers could endow them with multiple properties and functions, thereby presenting potential advantages in the context of smart materials. Thus, this review focuses on the fabrication strategies, interesting properties, and potential applications of fluorescent supramolecular polymers based on crown ethers. Typical examples are presented and discussed in terms of three different types of building blocks, namely covalently bonded low-molecular-weight compounds, polymers modified by hosts or guests, and supramolecular coordination complexes.

Keywords: supramolecular polymer, fluorescence, crown ether, host-guest interaction, supramolecular coordination complex

\section{INTRODUCTION}

Molecular self-assembly is the key to obtaining complicated biomolecules in natural systems, such as proteins, nucleic acids, phospholipid membranes, ribosomes, and microtubules (Chen et al., 2016; Laurent et al., 2017; Sun et al., 2019). Drawing inspiration from the vast number of assemblies present in nature, functional materials for applications in luminescent materials, sensors, bioimaging, and drug delivery (Chen L. -J. et al., 2015; Yan et al., 2015; Zhang M. et al., 2016; Yu et al., 2017; Zhang et al., 2017a) have been obtained through molecular self-assembly to form diverse supra-structures, such as micelles, vesicles, ribbons, and fibers (Ji et al., 2013a; Yan et al., 2013; Shi et al., 2016). In contrast to molecules based on traditional covalent bonds, supramolecular self-assembled structures exhibit specific characteristics, such as self-healing, coordinability, and responsiveness to stimuli, due to the dynamic and reversible nature of the non-covalent bonds or dynamic 


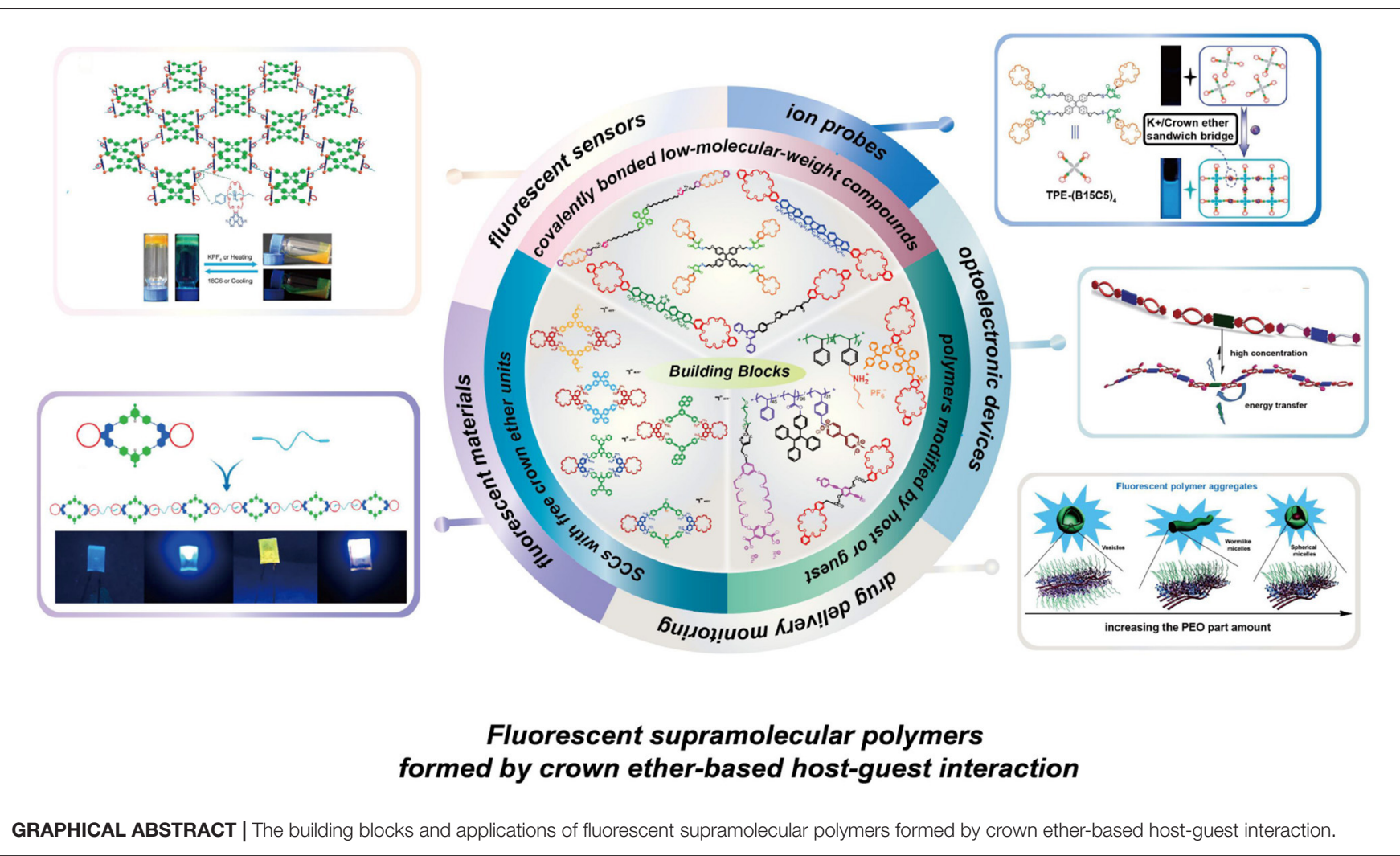

covalent bonds (Yan et al., 2012a; Yu et al., 2013, 2014; Zhang et al., 2018, 2019a; Deng et al., 2020). Among them, supramolecular polymers, in which repeating units are held together to form polymeric arrays through intermolecular bonds (e.g., host-guest interactions, hydrogen bonding, hydrophobic/hydrophilic interactions, metal-ligand interactions, and $\pi-\pi$ stacking) are considered to be promising smart materials.

To date, host-guest recognition systems have been widely employed to construct supramolecular polymers, where the hosts are often crown ethers, cyclodextrins, calixarenes, cucurbituril, pillararenes, and other macrocyclic hosts (Ma and Zhao, 2015; Qu et al., 2015; Yu et al., 2015; Liu et al., 2018; Shi et al., 2019). Among them, crown ethers, which were the first artificial macrocycles, are a type of macrocyclic polyether containing multiple oxygen methylene units, with examples including 18-crown-6, 21-crown-7, 24-crown-8, and other analogous derivatives, which can be host to positive ions and neutral molecules (Yamaguchi et al., 1998; Gibson et al., 2002; Huang and Gibson, 2004; Wei et al., 2015). In 1967, Pedersen reported, for the first time, stable complexes formed by crown ethers and certain cations that interacted via ion-dipole interactions between the cations and the high electron-density oxygen atoms of the crown ethers (Pedersen, 1967). Other studies have focused on the recognition between crown ethers and metal cations, such as $\mathrm{K}^{+}, \mathrm{Li}^{+}$, and $\mathrm{Na}^{+}$(Pedersen, 1967; Ma et al., 2015); however, in the wake of in-depth studies, complexes constructed from crown ethers and organic cations or organic neutral molecules were discovered, with examples including secondary ammonium salts, diazonium salts, and paraquat (Yamaguchi and Gibson, 1999; Gibson et al., 2003; Huang et al., 2007; Zhang et al., 2007). Crown ethers are known to accommodate a variety of guests, and they tend to exhibit strong binding affinities for specific guests due to their multidentate structure and relatively strong non-covalent interactions. Therefore, host-guest recognition systems based on crown ethers offer distinct advantages in terms of fabricating supramolecular polymers (Yan et al., 2012b; Zheng et al., 2012; Ding et al., 2013; Li X. et al., 2018; Li et al., 2019b; Xiao et al., 2020). Importantly, the formation of supramolecular polymers can overcome issues related to the preparation of traditional polymers, since the latter methods tend to require an auxiliary initiator, high temperatures, and long reaction times. Furthermore, the introduction of host-guest interactions can also endow the constructed supramolecular polymers with dynamic and reversible properties (Dong et al., 2012; Yan et al., 2014; Zhan et al., 2014a,b; Huang et al., 2018; Wang et al., 2020). For example, Dong and co-workers made a supramolecular polymer network with dynamic reversibility, good malleability, and processability that depended on the formation of host-guest interactions between crown ethers and ammonium motifs of H2G2-type monomer (Wang et al., 2020). Moreover, recently, a crown ether-based interaction has been used to enhance the mechanical strength of a supramolecualr polymer, which is promising for more exciting applications (Shi et al., 2020). Although a range of supramolecular polymers have been constructed based on host-guest interactions, 
the development of crown ether-based supramolecular polymers with additional multiple functionalities remains of interest.

Fluorescence refers to a cold luminescence phenomenon cause by photoluminescence, which is light emitted by a substance after it absorbs light or other electromagnetic radiation. In recent years, fluorescent materials have been widely used in life and material science. Fluorophores, which can emit fluorescence, are often incorporated into supramolecular polymers, since the resulting polymers inherit the fluorescence properties of the fluorogens in addition to exhibiting the dynamic and reversible properties originating from the noncovalent interactions. This renders them capable of exhibiting a fluorescence response to various external stimuli (Dong et al., 2016; Li et al., 2016, 2020; Wang et al., 2017). Fluorescent supramolecular polymers have therefore been widely applied in fluorescent materials, fluorescent probing, data storage, bio-imaging, drug delivery, and cancer therapy (Lou and Yang, 2018; Li et al., 2019a). Indeed, various kinds of organic fluorogens exist, such as coumarins, fluoresceins, cyanine naphthalimide rhodamine, conjugated polymer groups, and aggregation-induced emission (AIE) luminogens (Dsouza et al., 2011; Fermi et al., 2014; Ma et al., 2016; Peng et al., 2017; Li Y. et al., 2018). In addition, when conventional organic chromophores were combined with supramolecular polymers, the resulting polymers were found to exhibit weak fluorescence, since the formation of supramolecular polymers must be carried out at high concentrations, thereby causing aggregation-caused quenching (ACQ) of the traditional fluorescent chromophore. This issue can be efficiently resolved through the use of AIE luminogens, which were initially developed by Luo et al. (2001) and were found to exhibit faint luminescence in a dilute solution but strong luminescence in the solid or aggregate state due to the restriction of intramolecular vibrations and rotations. Furthermore, Lou and Yang focused on the combination of AIEgens with supramolecular macrocyclics, with a recent review of the supra-structures obtained, which included supramolecular nanocomplexes/polymers,
A
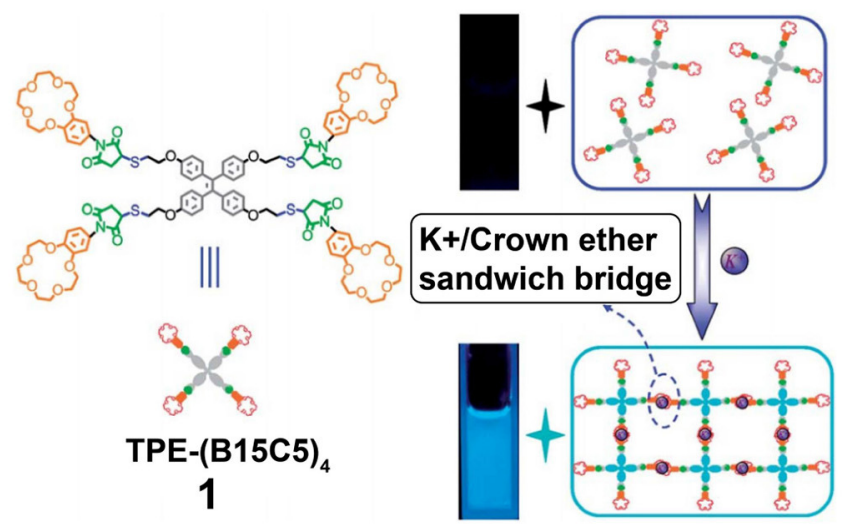

C
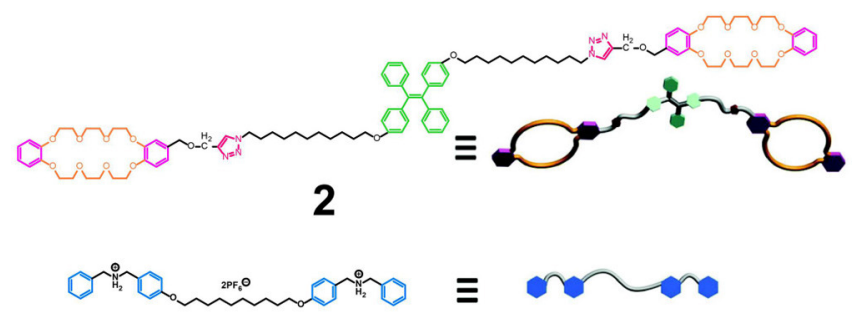

3

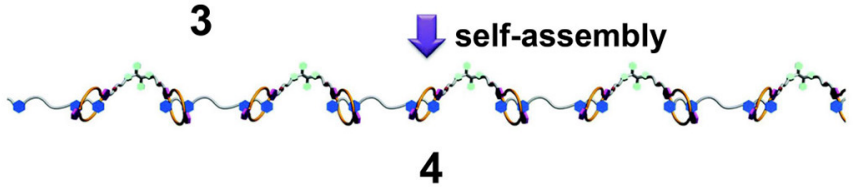

B

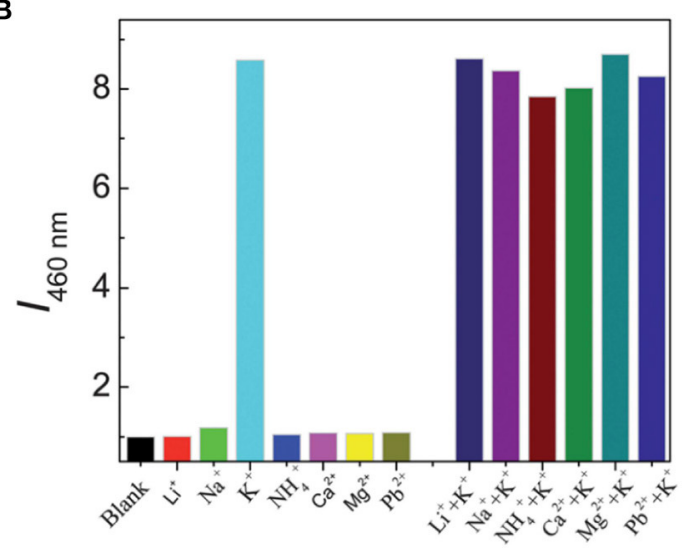

D

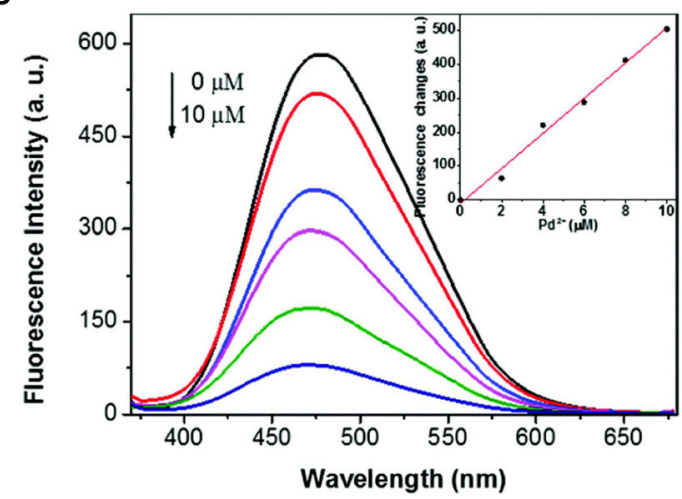

FIGURE 1 | (A) Schematic illustration of the AIE of TPE-(B15C5) 4 induced by recognition between the crown ether moieties and $\mathrm{K}^{+}$ions. (B) Relative fluorescence intensity at $\lambda_{\text {em }}=460 \mathrm{~nm}$ of TPE-(B15C5) 4 in THF solution recorded under the addition of $\mathrm{K}^{+}$ions in the absence or presence of other interfering ions (i.e., $\mathrm{Li}^{+}$, $\mathrm{Na}^{+}$, $\mathrm{NH}_{4}^{+}, \mathrm{Ca}^{2+}, \mathrm{Mg}^{2+}$, and $\left.\mathrm{Pb}^{2+}\right)\left(\lambda_{\mathrm{ex}}=360 \mathrm{~nm}\right.$; slit width: Ex. $5 \mathrm{~nm}$, Em. $5 \mathrm{~nm} ; 25^{\circ} \mathrm{C}$ ). Adapted with permission from Wang et al. (2012); copyright 2012, Royal Society of Chemistry. (C) Cartoon representations of the formation of the linear fluorescent supramolecular polymer $\mathbf{4}$ from host $\mathbf{2}$ and guest $\mathbf{3}$. (D) Fluorescence emission spectra of $\mathbf{4}$ with different concentrations of $\mathrm{Pd}^{2+}(0-10 \mu \mathrm{M})$ in the solid state $\left(\lambda_{\mathrm{ex}}=350 \mathrm{~nm}\right)$. Inset: Linear relationship between the fluorescence intensity of $\mathbf{4}$ and the concentration of $\mathrm{Pd}^{2+}$ at $\lambda_{\mathrm{em}}=577 \mathrm{~nm}$. Adapted with permission from Chen D. et al. (2015); copyright 2015, Royal Society of Chemistry. 
supramolecular nanoparticles, and host-guest complexes on nanosurfaces (Lou and Yang, 2018). Thus, in the next parts of this review, we will focus on fluorescent supramolecular polymers based on crown ethers, which are not confined to AIE-active luminogens, and discuss their fabrication strategies, interesting properties, and potential applications. In addition, we present diverse methods for combining free crown ether units with various building blocks, including covalently bonded low-molecular-weight compounds, polymers modified by hosts or guests, and supramolecular coordination complexes (SCCs), and representative examples are scrutinized over a comprehensive scope.

\section{CROWN ETHER-BASED FLUORESCENT SUPRAMOLECULAR POLYMERS CONSTRUCTED BY COVALENTLY BONDED LOW-MOLECULAR-WEIGHT COMPOUNDS}

The most efficient and straightforward method of constructing fluorescent supramolecular polymers is to design and prepare fluorescent hosts or guests through the rational chemical modification of chromophores. Subsequently, certain crown ethers and guests are brought together through host-guest<smiles></smiles>
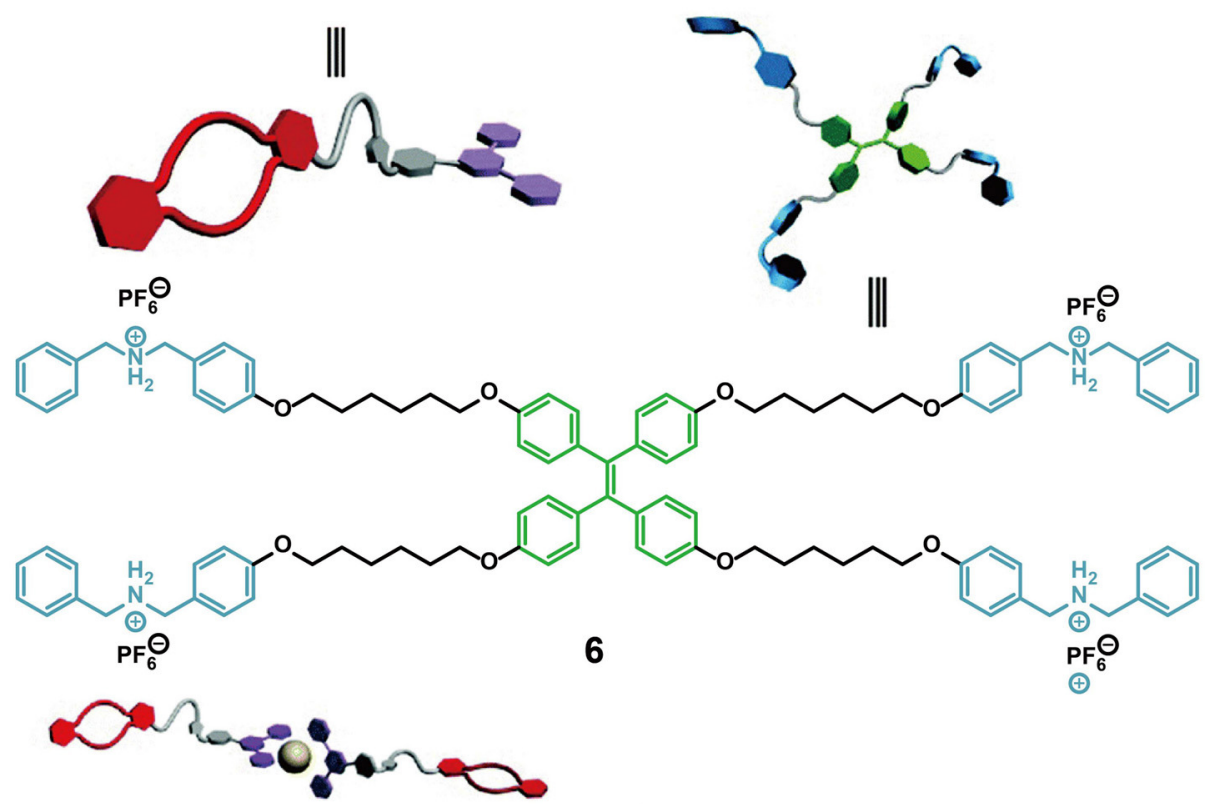

Supramolecular Dimer 8

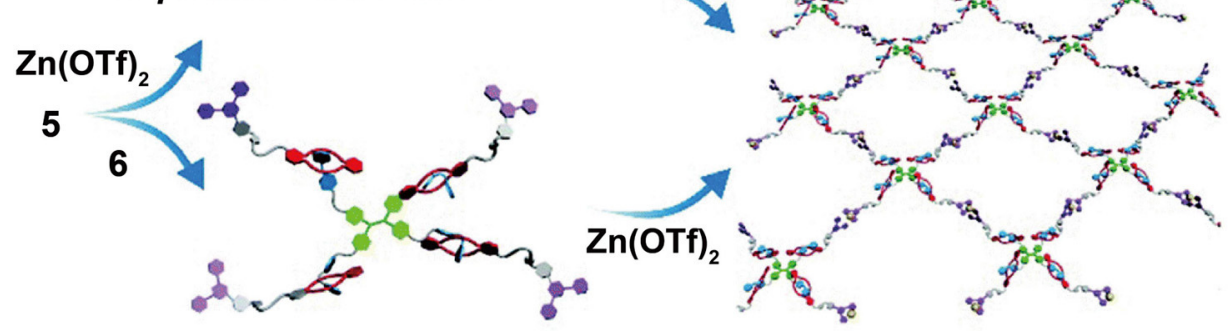

Supramolecular Tetramer 9 Hyperbranched Supramolecular Polymer 7

FIGURE 2 | Schematic illustration of monomers $\mathbf{5}$ and $\mathbf{6}$ and cartoon representations of the formation of supramolecular dimer $\mathbf{8}$, supramolecular tetramer $\mathbf{9}$, and hyperbranched supramolecular polymer 7. Adapted with permission from Zhang J. et al. (2016); copyright 2016, Royal Society of Chemistry. 
interactions along with $\pi-\pi$ stacking (Ma et al., 2015) and/or donor-acceptor interactions (Roy et al., 2016).

More specifically, Wang et al. reported the application of AIE-active supramolecular polymers based on the recognition system of crown ethers (Wang et al., 2012). They designed a novel and effective fluorometric $\mathrm{K}^{+}$probe via host-guest molecular recognition and aggregation-induced emission. The host molecule TPE-(B15C5) 4 (1) was synthesized by the functionalization of a four peripheral benzo-15-crown-5 (B15C5) with tetraphenylethylene (TPE) as the core (Figure 1A). The AIE feature of the TPE cores and the formation of crosslinked supramolecular polymers resulted in the aggregation of 1 and an enhancement in fluorescence emission; this system was suitable for application in $\mathrm{K}^{+}$detection. Moreover, 1 exhibited an excellent selectivity toward $\mathrm{K}^{+}$compared to other interfering ions (e.g., $\mathrm{Li}^{+}, \mathrm{Na}^{+}, \mathrm{NH}_{4}^{+}, \mathrm{Ca}^{+}, \mathrm{Mg}^{+}$, and $\mathrm{Pd}^{2+}$ ) (Figure 1B), with a detection limit of $\sim 1.0 \mu \mathrm{M}$. This work opened a new avenue for the development of sensitive and selective fluorometric off-on probes. Similarly, Chen D. et al. (2015) designed monomer 2, wherein the host TPE core was linked with two dibenzo-24-crown-8 (DB24C8) and two 1,2,3-trizole units, and a dibenzylammonium (DBA) salt monomer 3 was employed as the guest. Consequently, an AIE-active supramolecular polymer 4 (Figure 1C) was obtained through host-guest interactions by mixing monomers 2 and 3 in a 1:1 molar ratio. The characteristic AIE properties of supramolecular polymer $\mathbf{4}$ originated from restriction of the TPE group intramolecular rotational motion. These properties were confirmed by observation that the fluorescence intensity of 4 was 6-fold stronger than that of monomer 1 at the same concentration. In addition, the enhanced fluorescence intensity of 4 was accompanied by a 13-nm red-shift upon increasing its concentration from 75 to $200 \mathrm{mM}$. Furthermore, the fluorescence intensity of $\mathbf{4}$ in the solid state was further increased along with a 79-nm blue-shift due to morphological changes in the aggregates. Interestingly, the fluorescence intensity of $\mathbf{4}$ decreased linearly

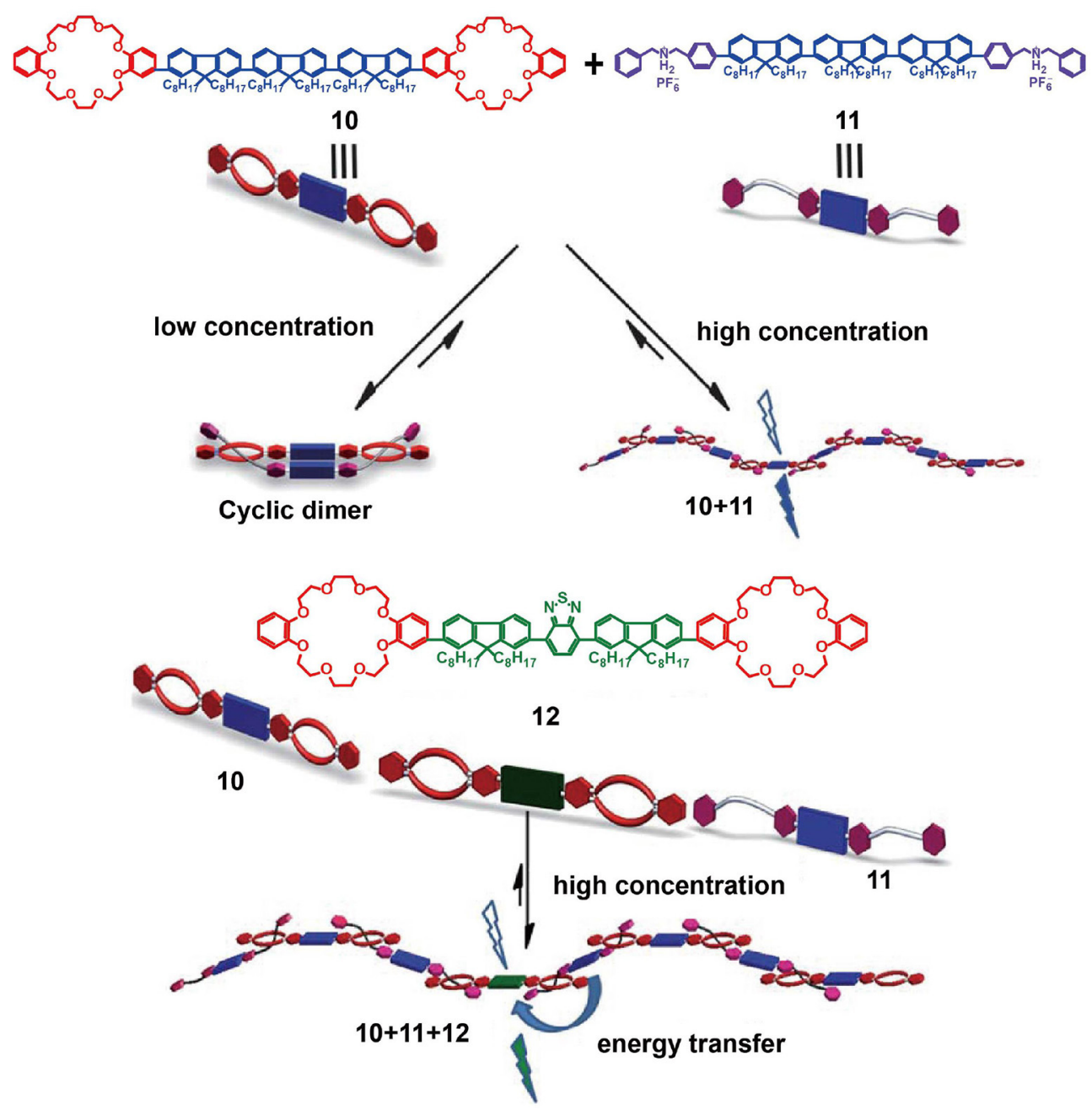

FIGURE 3 | Cartoon representations of the formation of SLEPs from host 10, guest 11, and doping host 12. Adapted with permission from Zhang et al. (2012); copyright 2012, Royal Society of Chemistry. 
upon the addition of $\mathrm{Pd}^{2+}$ (Figure 1D) owing to coordination interactions and energy transfer between the 1,2,3-trizole units and $\mathrm{Pd}^{2+}$. Thus, 4 can be employed as a $\mathrm{Pd}^{2+}$ fluorescent probe in the solid state, thereby enriching the application of supramolecular polymeric materials.
In addition to their application as ion sensors, crown etherbased fluorescent supramolecular polymers formed using TPEfunctionalized monomers can also be employed as smart and adaptive luminescent materials. For example, Zhang J. et al. (2016) designed two monomers, one (5) with a DB24C8 group
A
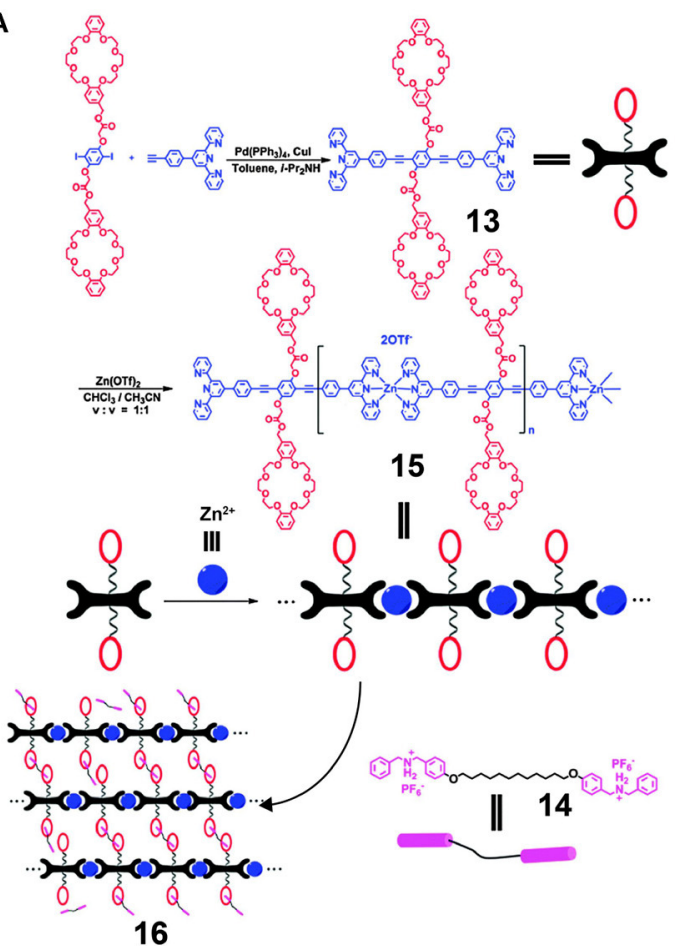

B

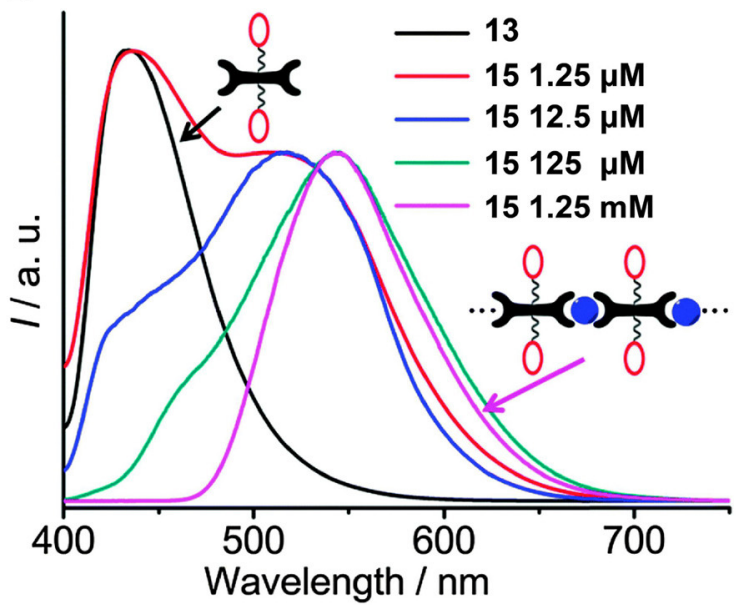

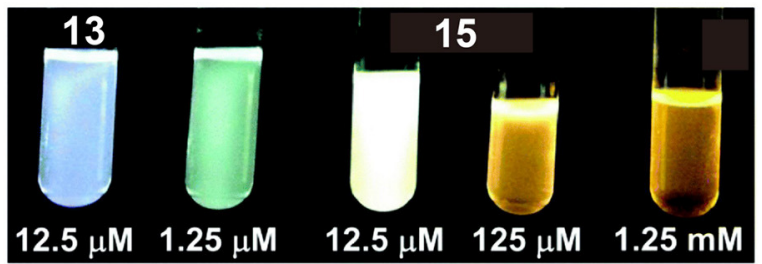

FIGURE 4 | (A) Synthetic approaches and cartoon representations of the formation of $\mathbf{1 5}$ and $\mathbf{1 6}$. (B) Fluorescence emission spectra of $\mathbf{1 3}$ and $\mathbf{1 5}$ in $\mathrm{CHCl}_{3} / \mathrm{CH}_{3} \mathrm{CN}$ $(v / V=1: 1)$ and the corresponding optical photographs recorded under UV light $\left(\lambda_{\mathrm{ex}}=365 \mathrm{~nm}\right)$. Adapted with permission from He et al. (2014); copyright 2015, Royal Society of Chemistry.

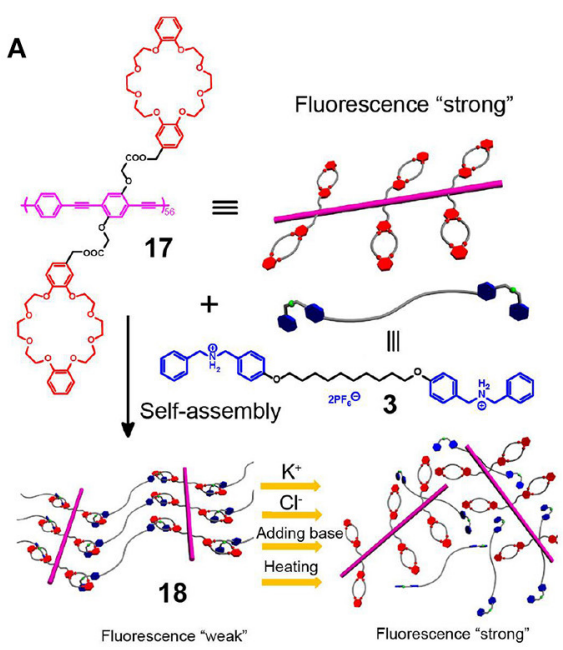

\section{B}

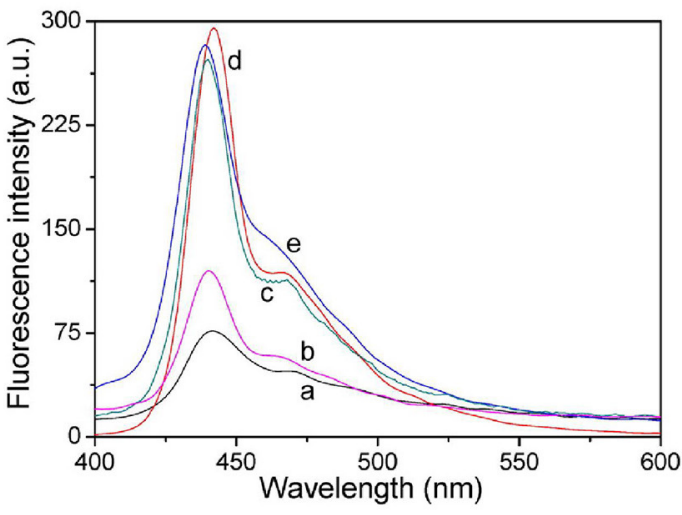

FIGURE 5 | (A) Cartoon representations of the formation of the supramolecular cross-linked network $\mathbf{1 8}$ from polymer $\mathbf{1 7}$ and cross-linker $\mathbf{3}$. (B) Fluorescence emission spectra of (a) $\mathbf{1 8}$ constructed using $\mathbf{1 7}(2.0 \mu \mathrm{M})$ and $\mathbf{3}(100 \mu \mathrm{M})$ and after (b) treatment by heating at $50^{\circ} \mathrm{C}$ and treatment with (c) $200 \mu \mathrm{M} \mathrm{KPF} 6$, (d) $200 \mu \mathrm{M}$ TBACl, and (e) $200 \mu \mathrm{M} \mathrm{Et}_{3} \mathrm{~N}$. Adapted with permission from Ji et al. (2013b); copyright 2013, American Chemical Society. 
at one end and a terpyridine moiety at the other end, and another (6) with a TPE core and four outer DBA salts. A hyperbranched fluorescent supramolecular polymer, 7, was constructed through the connection of $\mathrm{Zn}(\mathrm{OTf})_{2}$ with monomers 5 and $\mathbf{6}$ via terpyridine-based metal-ligand interactions and crown etherbased host-guest interactions (Figure 2). The hyperbranched fluorescent supramolecular polymer 7 displayed a strong emission, while supramolecular dimer $\mathbf{8}$ and supramolecular tetramer $\mathbf{9}$, which were formed by only one kind of non-covalent interaction, exhibited a reduced luminescence due to inefficient restriction of the intramolecular rotation/torsion of the TPE groups in $\mathbf{8}$ and 9. In addition, 7 exhibited responsiveness to multiple stimuli, including temperature, $\mathrm{pH}, \mathrm{K}^{+}$, and $\mathrm{Cl}^{-}$, due to the dynamic and reversible nature of the two orthogonal noncovalent interactions. Coincidentally, a further $\mathrm{pH}$-responsive supramolecular polymer was fabricated by Bai et al. (2015) based on a similar principle, whereby the polymer consisted of TPE entities bearing DB24C8 or DBA on opposite phenyl rings. These results therefore provide a representative demonstration of self-assembly-induced emission (SAIE), which is conducive to the development of novel supramolecular materials exhibiting a stimulus-responsive fluorescence transition.

In addition to the introduction of AIEgens such as TPE as luminescent groups, the use of fluorescent conjugated oligomers would also present potential advantages in the development of optoelectronic devices or fluorescence materials. For example, Zhang et al. (2012) reported supramolecular light-emitting polymers (SLEPs) prepared from blue-emitting conjugated oligomer $\mathbf{1 0}$ and green-emitting conjugated oligomer $\mathbf{1 2}$ as the hosts and blue-emitting conjugated oligomer $\mathbf{1 1}$ as the guest (Figure 3). The resulting polymer, based on host-guest interactions, exhibited good film formation abilities, a stable film morphology, and facile solution processability. Furthermore, the polymer emission was tuneable by controlling the content of dopant host 12. More specifically, by doping 10 or $30 \%$ of 12 into the supramolecular system to promote efficient energy transfer among the oligomers, the resulting SLEPs showed a large red-shift photoluminescent emission, significantly enhanced photoluminescent efficiencies, and achieved superior device performance. Due to these advantages, SLEPs have the potential to promote the development of solution-processed optoelectronic devices.

To endow supramolecular polymers with more versatile topological structures and increase their suitability for practical applications, $\mathrm{He}$ et al. (2014) constructed a cross-linked metallosupramolecular polymer, $\mathbf{1 6}$, by employing metalligand interactions between conjugated bis-terpyridine ligand 13 and $\mathrm{Zn}^{2+}$ as the chromophore in addition to hostguest interactions between the two DB24C8 moieties in $\mathbf{1 3}$ and the two DBA groups in 14 (Figure 4A). The resulting linear conjugated supramolecular polymer 15 based on the terpyridine/Zn recognition motifs exhibited concentrationcontrollable emission varying in color from cyan to white to yellow (Figure 4B) due to the presence of components

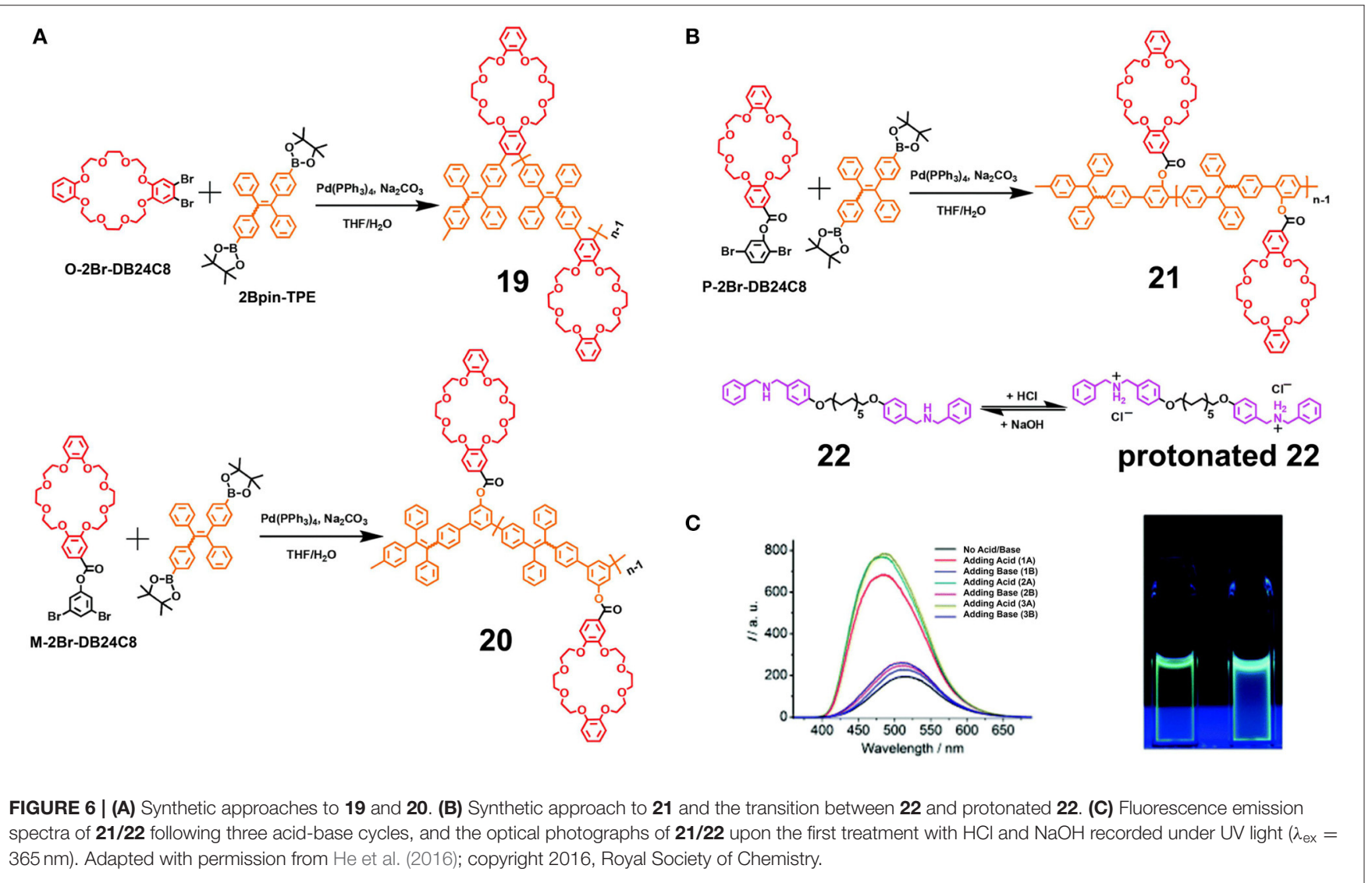


bearing different numbers of repeat units, including monomers, oligomers, and polymers. Interestingly, when the concentration of $\mathbf{1 5}$ is $12.5 \mu \mathrm{M}$, nearly white emission occurred in the absence of other complementary fluorescence groups. Moreover, upon increasing the quantities of added guest molecules 14 into 15, the fluorescence intensity decreased gradually due to the formation of cross-linked metallosupramolecular polymer 16 via hostguest interactions between the DB24C8 moieties and the DBA moieties. In addition, the fluorescence intensity of 16 varied with changes in $\mathrm{pH}$, which renders $\mathbf{1 6}$ suitable for application in acid-base stimulus-responsive materials. With such dual responsiveness, this supramolecular polymer represents an important advance in the design of fluorescent materials and molecular devices.

\section{CROWN ETHER-BASED FLUORESCENT SUPRAMOLECULAR POLYMERS CONSTRUCTED USING POLYMERS}

The construction of crown ether-based fluorescent supramolecular polymers using covalently bonded low-molecular-weight compounds has a number of advantages, including simple synthetic routes, clear molecular structures, and ease of building supramolecular polymers with complicated topological structures. However, the stabilities and mechanical properties of the resulting supramolecular polymers tend to be poor, and so to overcome this issue, the incorporation of covalently bonded polymers into the supramolecular polymeric systems is an option. Indeed, with the development of supramolecular chemistry and polymer science, growing numbers of tailor-made covalent polymers have been employed as building blocks to construct supramolecular polymers through non-covalent interactions, and these polymers inherit the properties of both covalent and non-covalent bonding, including the associated mechanical properties, photophysical properties, reversibility, and stimuli-responsiveness. In such cases, polymers can play the roles of the host, the guest, or both.

\section{Polymers as Hosts and Micromolecules as Guests}

In the case where polymers are employed as hosts and micromolecules as guests, crown ethers are modified into the traditional polymer hosts, while low-molecular-weight secondary ammonium salts, diazonium, salts, and paraquat are used as the guests. More specifically, Ji et al. (2013b) developed a supramolecular cross-linked network 18 via hostguest interactions through the use of fluorescent conjugated polymer chains grafted with the DB24C8 groups of $\mathbf{1 7}$ as the host and a bisammonium salt as the guest (3) (Figure 5A). Compared with the fluorescence intensity of polymer 17, that of the supramolecular cross-linked network $\mathbf{1 8}$ was significantly lower due to the ACQ properties of the conjugated poly(phenylene ethynylene) polymeric backbones. The structure of polymer network 18 could be destroyed by multiple stimuli, such as variations in the temperature or $\mathrm{pH}$ change or the addition of $\mathrm{K}^{+}$or $\mathrm{Cl}^{-}$ions, and this was accompanied by an increase in the fluorescence intensity due to the presence of dynamic non-covalent bonds (Figure 5B). Interestingly, the cross-linked polymer network thin film emitted a stronger fluorescence upon exposure to an alkaline gas, such as ammonia vapor, since the deprotonation of cross-linker 3 resulted in dissociation of the aggregated state of the poly(phenylene ethynylene) polymer main chains. Hence, this system could be employed to probe various stimuli, and in particular, the presence of an alkaline gas, and so can be considered an attractive candidate for advanced sensor materials.

To overcome the weak fluorescence exhibited by traditional conjugated polymers upon aggregation, $\mathrm{He}$ et al. (2016) constructed a series of crown ether-based fluorescent polymers 19-21 with AIE properties by coupling 2Bpin-TPE as an AIEgen with $\mathrm{O}-2 \mathrm{Br}-\mathrm{DB} 24 \mathrm{C} 8, \mathrm{M}-2 \mathrm{Br}-\mathrm{DB} 24 \mathrm{C} 8$, and $\mathrm{P}-2 \mathrm{Br}-\mathrm{DB} 24 \mathrm{C} 8$, in addition to a bisammonium salt 22 (Figures 6A,B). It should be noted here that the structural difference between polymers 19-21 was the linkage position of the TPE group. Thus, polymers 1921 exhibited different degrees of aggregation-induced emission enhancement (AIEE) upon increasing the fraction of the poor solvent present in the mixture. For example, 19 exhibited a relatively bright emission in the dilute solution state, while its fluorescence enhancement was limited upon aggregation. This

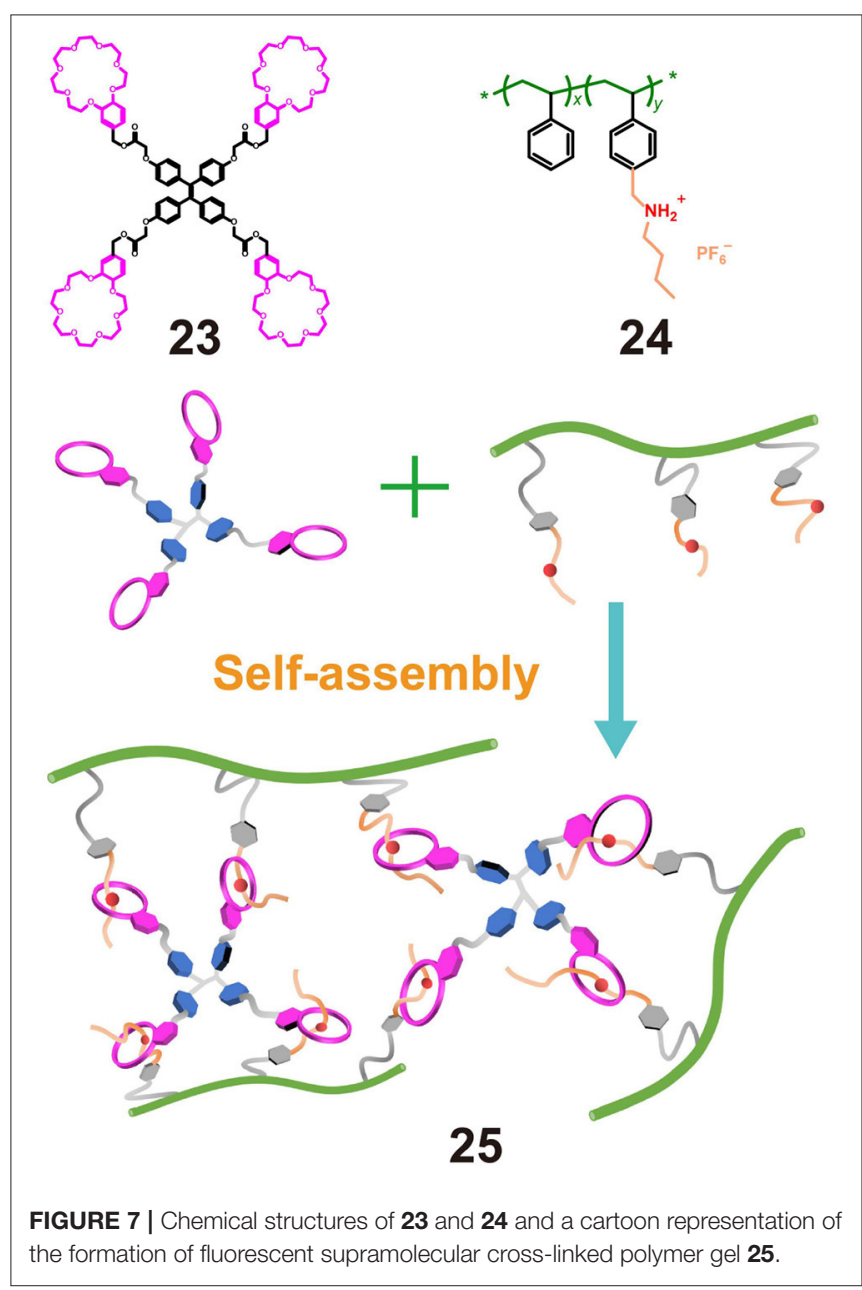


was accounted for by considering that the suppression of the intramolecular motion of the TPE moieties in $\mathbf{1 9}$ connected at the ortho-position was more efficient than the associated suppression in $\mathbf{2 0}$ at the meta-position and in $\mathbf{2 1}$ at the para-position. Interestingly, following treatment with three acid-base cycles, the system formed using $\mathbf{2 1}$ and $\mathbf{2 2}$ exhibited a highly reversible fluorescence intensity and emission wavelength (Figure 6C), while the systems formed from $\mathbf{1 9}$ and $\mathbf{2 2}$ or $\mathbf{2 0}$ and $\mathbf{2 2}$ exhibited stepwise increases in their fluorescence intensities. This was attributed to recognition between DB24C8 and DBAS and the efficient salting-out effect of $\mathrm{NaCl}$ generated from the acidbase process, which in turn facilitated morphological evolution from micelles to larger vesicles. However, due to the more rigid conformation of the polymer chain of $\mathbf{2 1}$, the construction of vesicles from the $\mathbf{2 1 / 2 2}$ system was more challenging, and so stabilization of the fluorescence intensity was less efficient. Based on such findings, these polymers can be considered promising materials for use in the field of optoelectronic devices and fluorescent sensors.

\section{Polymers as Guests and Micromolecules as Hosts}

In the case were polymers are employed as guests and micromolecules as hosts, secondary ammonium salts are commonly hung on traditional polymers to make the guest, while low-molecular-weight crown ethers are employed as the hosts. For example, Ji X. F. et al. (2015) synthesized polystyrene polymer $\mathbf{2 4}$ bearing dialkylammonium salt moieties as pendent groups and benzo-21-crown-7 (B21C7) macrocycles 23 functionalized on the four arms of TPE molecules. Upon mixing 24 and 23, a fluorescent supramolecular polymer gel was obtained via host-guest interactions between the B21C7 units and the dialkylammonium salts (Figure 7). The gel emitted a strong fluorescence, while a solution of 23 containing the same molar concentration of TPE units showed almost no fluorescence, thereby indicating that gelation induced the fluorescence emission. In addition, the presence of non-covalent interactions rendered the gel responsive to changes in temperature and $\mathrm{pH}$. Thus, upon heating or with the addition of triethylamine, the recognition between $\mathrm{B} 21 \mathrm{C} 7$ and the dialkylammonium salts was destroyed, resulting in disassembly of the gel and the formation of a sol, which was accompanied by fluorescence quenching. However, the recognition between B21C7 and the dialkylammonium salts was easily recovered by cooling or by the addition of trifluoroacetic acid. This work therefore provided a strategy for the construction of functional supramolecular polymers.

Employing the above-mentioned method, Xu et al. (2018a) prepared a polystyrene polymer $\mathbf{2 6}$ bearing coumarin moieties and dialkylammonium salts and combined this with a TPE derivative 27 bearing a DB24C8 unit and a terpyridine moiety at each side. A supramolecular network was constructed by the simple mixing of 26, 27, and $\mathrm{Zn}(\mathrm{OTf})_{2}$ through DB24C8/dialkylammonium salt and terpyridine/Zn recognition moieties (Figure 8A). Upon increasing the concentration of the
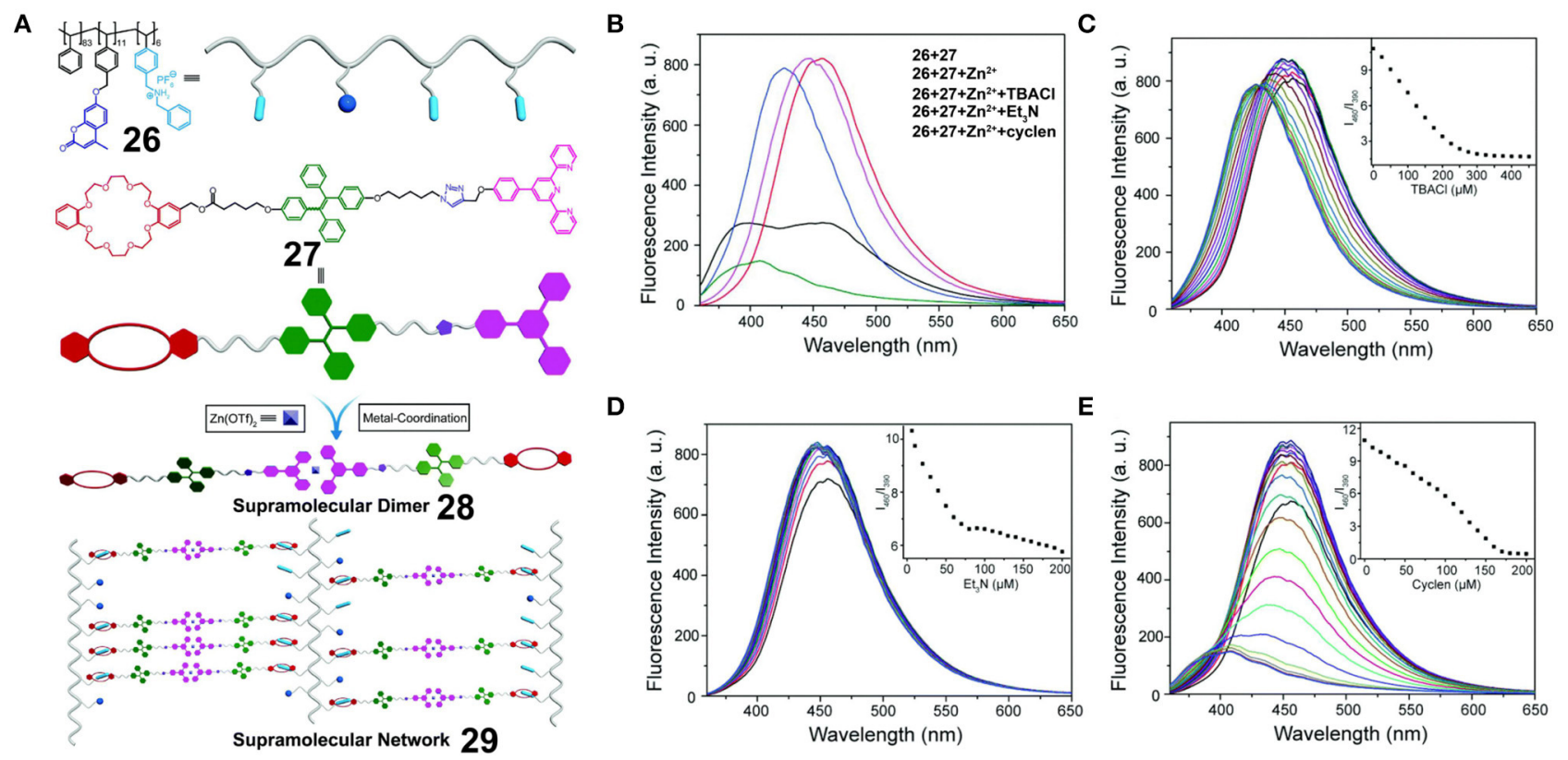

D

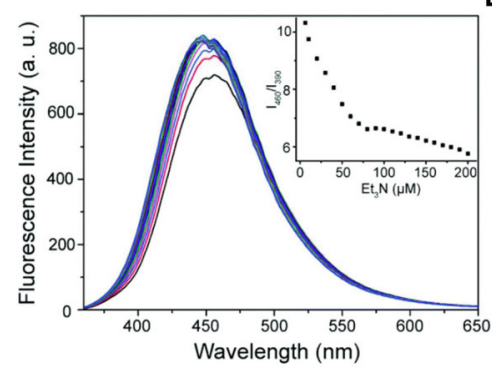

E

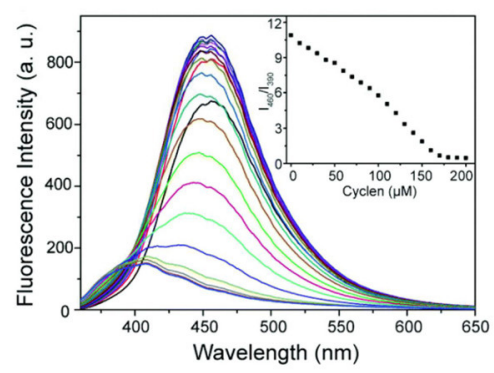

FIGURE $\mathbf{8}$ | (A) Chemical structures of $\mathbf{2 6}$ and $\mathbf{2 7}$, and cartoon representations of the formation of supramolecular dimer $\mathbf{2 8}$ and fluorescent supramolecular cross-linked polymer 29. (B) Fluorescence emission spectra of the mixture of $\mathbf{2 6}(18.6 \mu \mathrm{M})$ and $\mathbf{2 7}(100 \mu \mathrm{M})$ and upon the addition of Zn(OTf) 2 (50 $\mu \mathrm{M})$, TBACl $(450 \mu \mathrm{M}), \mathrm{Et}_{3} \mathrm{~N}(200 \mu \mathrm{M})$, or cyclen $(200 \mu \mathrm{M})$. Fluorescence emission spectra of the network upon the stepwise addition of (C) TBACl, (D) Et ${ }_{3} \mathrm{~N}$, and (E) cyclen. The insets show the plots of $I_{460} / /_{390}$ vs. the amount of (C) TBACl, (D) Et 3 N, and (E) cyclen added. Adapted with permission from Xu et al. (2018a); copyright 2018, Royal Society of Chemistry. 
system, the emission intensity of the supramolecular polymer network 29 at $460 \mathrm{~nm}$ (originating from the TPE units) was found to increase, while that at $390 \mathrm{~nm}$ (originating from the coumarin units) was found to decrease due to the AIE property of the TPE units and the ACQ property of the coumarin units. In this case, no obvious Förster resonance energy transfer (FRET) took place between $\mathbf{2 6}$ and $\mathbf{2 7}$ due to a lack of spectral overlap. Furthermore, the ratio between the emission intensities at 460 and $390 \mathrm{~nm}\left(I_{460} / I_{390}\right)$ varied linearly within a certain range upon the dissociation of 29 when $\mathrm{NEt}_{3}, \mathrm{Cl}^{-}$, or cyclen was added. Thus, the supramolecular polymer network 29 could be employed as a ratiometric sensor for $\mathrm{pH}$, cyclen, and $\mathrm{Cl}^{-}$with precise results (Figures 8B-E). Upon further increasing the concentration of the supramolecular polymer network 29, a cross-linked supramolecular gel exhibiting multiple stimulus responsiveness and self-healing behavior was formed. Based on the above results, this fluorescent supramolecular polymer provided a representative illustration of a fluorescent material to serve as a ratiometric sensor, whereby monitoring was possible through self-calibration from two emission peaks.

$\mathrm{Fu}$ et al. (2019) also prepared polystyrene 31 containing DBAS units as pendant groups. In addition, they synthesized an anthracene-bridged divalent crown ether 30 (Figure 9A) in which anthracene adopted the cis-conformation to prevent $\pi-\pi$ stacking between the anthracene units. Upon the simple mixing of $\mathbf{3 0}$ and $\mathbf{3 1}$ at an appropriately high concentration, a fluorescent supramolecular polymer gel formed based on DB24C8/DBAS recognition, and this polymer displayed a reversible gel-sol transition upon the addition of competitive guest or through $\mathrm{pH}$ or thermal stimuli, due to the nature of the dynamic and reversible non-covalent bond (Figures 9C,D). Furthermore, this luminescent supramolecular polymer gel possessed an attractive photo-controlled property due to the fact that host 30, which contains two bulky groups in the 9 and 10 positions of the anthracene skeleton, could react with singlet oxygen through a $[4+2]$ cycloaddition reaction upon irradiation with UV light, therefore leading to a decrease in fluorescence (Figure 9B).

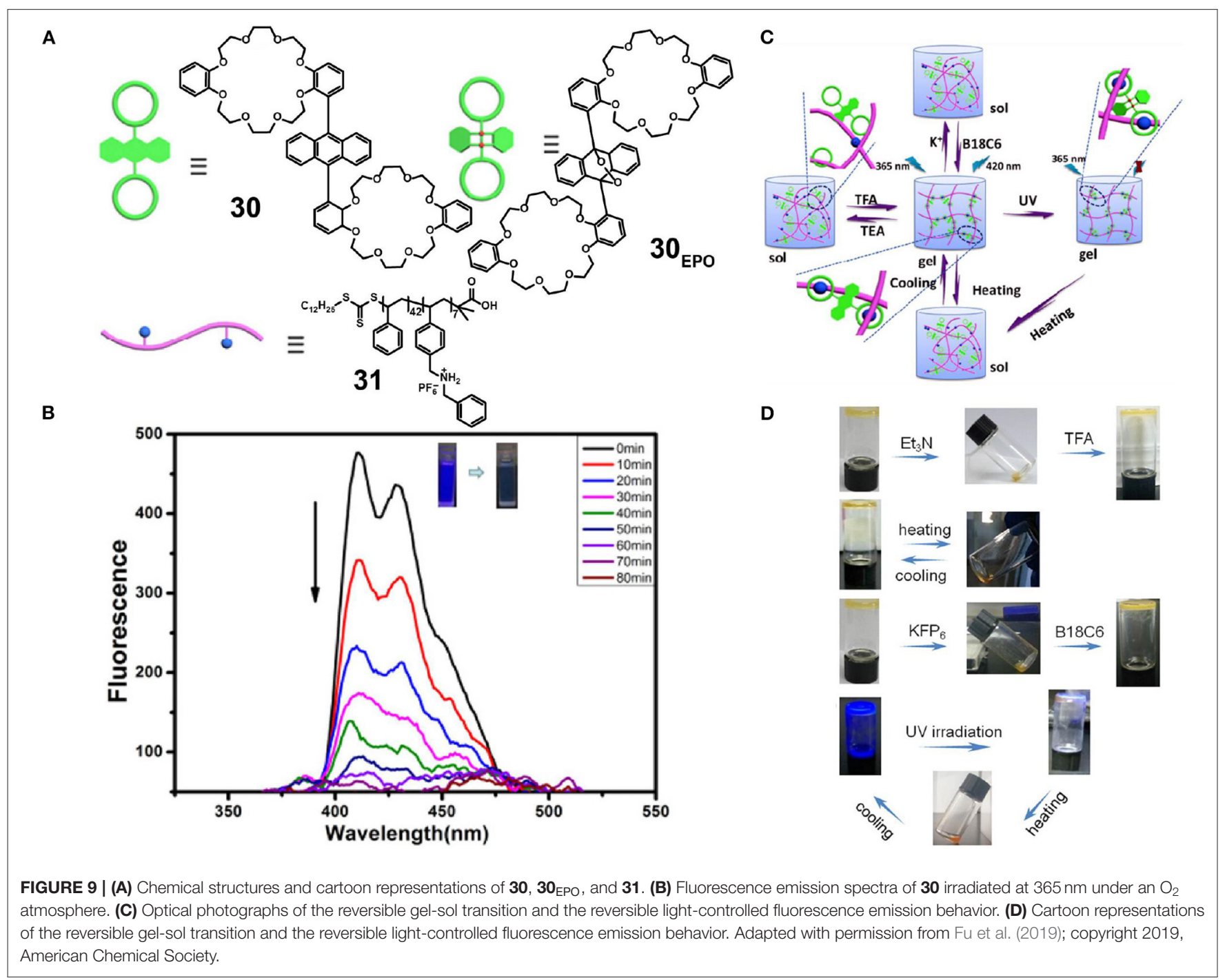




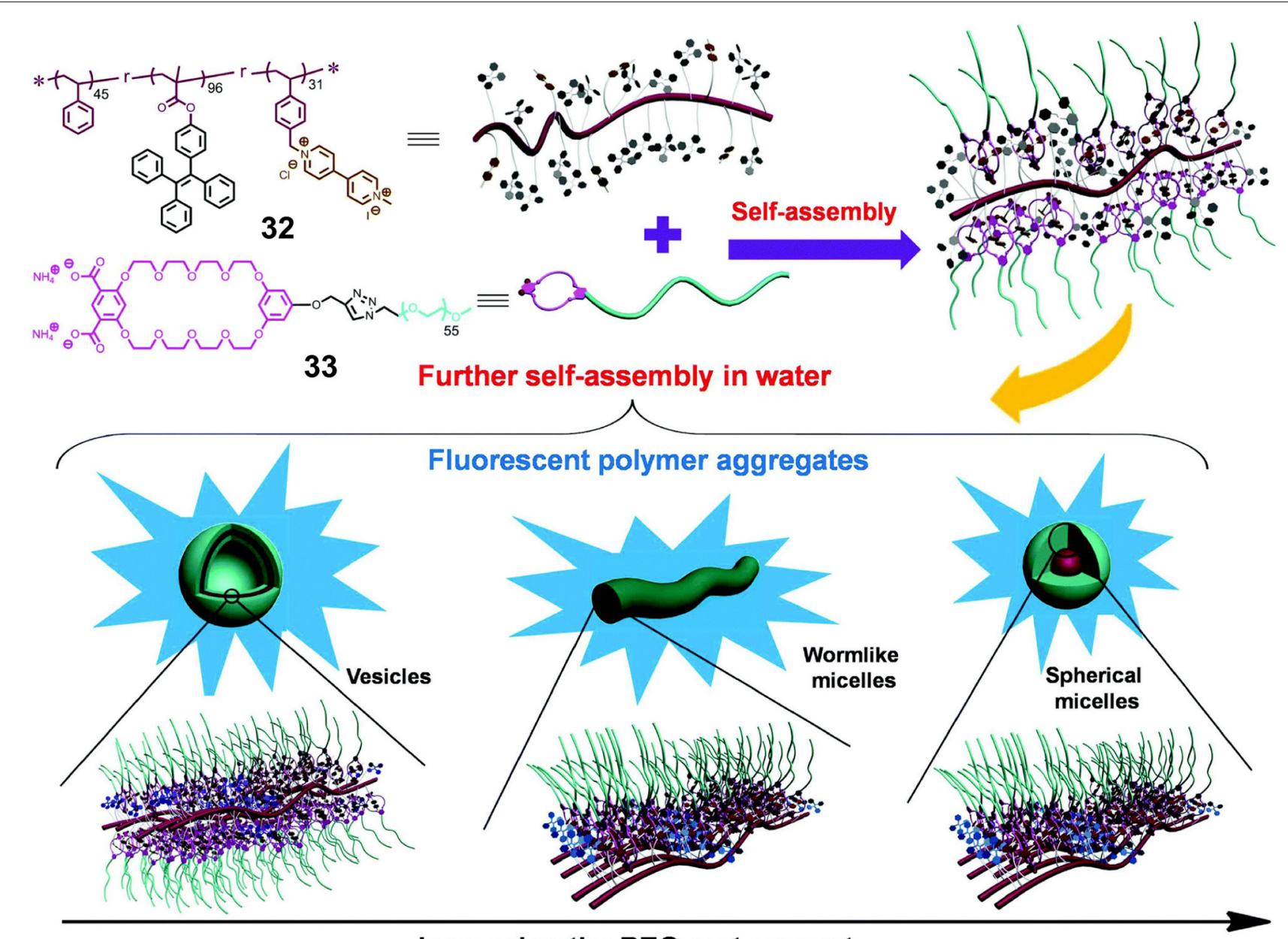

Increasing the PEO part amount

FIGURE 10 | Chemical structures and cartoon representations of $\mathbf{3 2}$ and $\mathbf{3 3}$, in addition to cartoon representations of supramolecular graft copolymer construction and further self-assembly into different morphologies. Adapted with permission from Ji X. et al. (2015); copyright 2015, Royal Society of Chemistry.
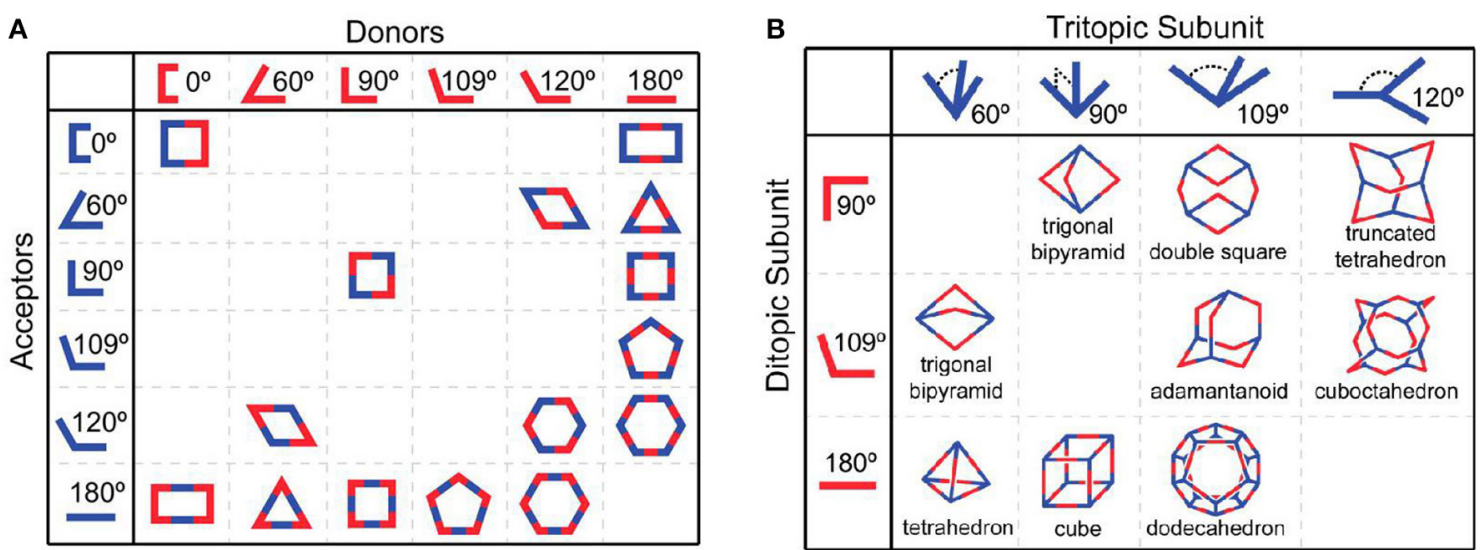

FIGURE 11 | The combination of different building blocks results in (A) 2D polygons and (B) 3D polygons. Adapted with permission from Cook et al. (2013); copyright 2013, American Chemical Society. 
This supramolecular system exhibits potential for application in fluorescent materials and, in particular, photo-controlled sensors.

\section{Polymers as Both Hosts and Guests}

In the case where polymers are employed both as the hosts and the guests, crown ethers and secondary ammonium salts are both employed to modify traditional polymers. In this context, Ji X. et al. (2015) investigated the influence of the aggregation morphology on the functions of fluorescent polymeric aggregation through constructing supramolecular systems based on hydrophobic polymer $\mathbf{3 2}$ and hydrophilic polymer 33 in water. More specifically, polymer 32 consisted of polystyrene with pendent TPE moieties and paraquat units, while polymer 33 consisted of a water-soluble poly(ethylene oxide) (PEO) terminated with a bis-(m-phenylene)-32-crown-10 (BMP32C10) unit containing two $\mathrm{COO}^{-}$groups. Unlike traditional intricate methods for the construction of fluorescent polymeric aggregates through the synthesis of a series of polymers, host-guest interactions between the BMP32C10 and paraquat units in $\mathbf{3 2}$ and 33 resulted in the formation of supramolecular amphiphilic graft copolymers. By adjusting the proportion of hydrophilic polymer 33, different aggregation morphologies, such as vesicles, wormlike micelles, and spherical micelles, can be formed (Figure 10). Thus, due to their amphipathy and special morphology, these supramolecular graft copolymers could encapsulate non-fluorescent drugs and subsequently release these drugs with the appropriate $\mathrm{pH}$ stimulus due to the stimulus responsiveness of $\mathrm{BMP} 32 \mathrm{C} 10 /$ paraquat recognition. This could be detected by changes in the intensity of fluorescence. Furthermore, due to the low cytotoxicities of $\mathbf{3 2}$ and $\mathbf{3 3}$ and the resulting polymer aggregates, this supramolecular system shows promise for application in the field of drug delivery.

\section{CROWN ETHER-BASED FLUORESCENT SUPRAMOLECULAR POLYMERS CONSTRUCTED BY SCCs}

In addition to the aforementioned strategies, discrete supramolecular coordination complexes (SCCs) with welldefined sizes, shapes, and geometries have been proven to be rational building blocks for the construction of fluorescent

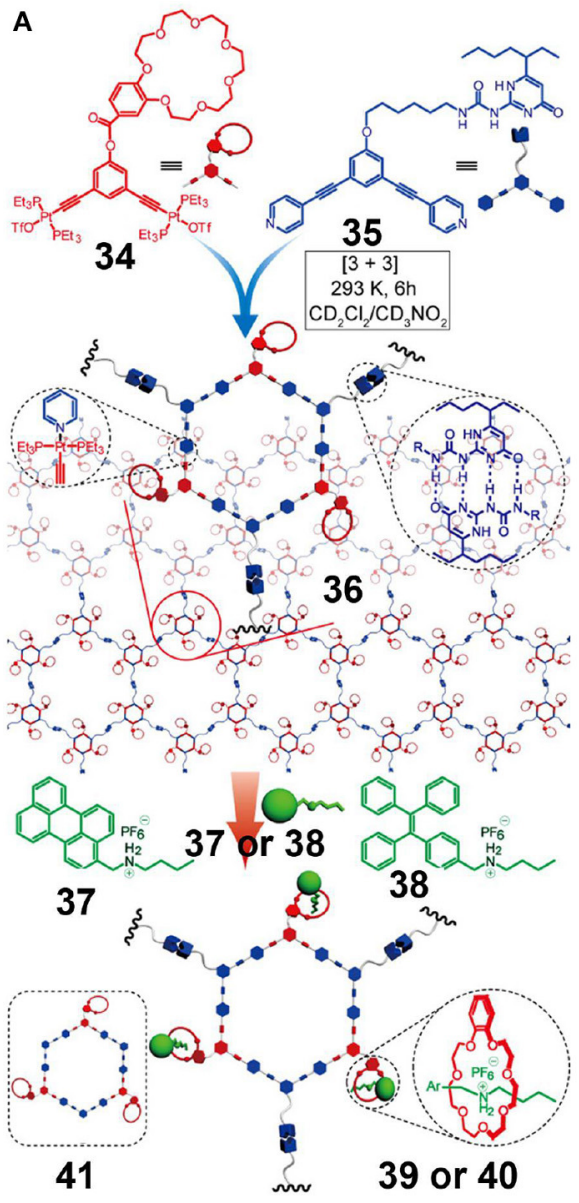

B
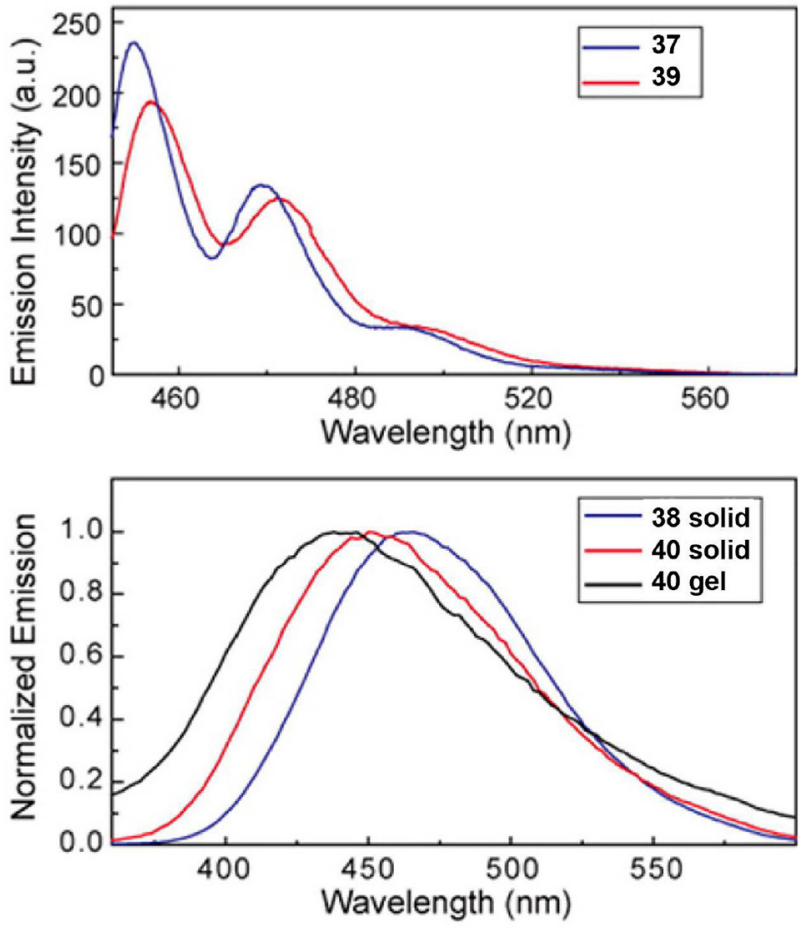

FIGURE 12 | (A) Chemical structures of platinum acceptor $\mathbf{3 4}$ and dipyridyl donor $\mathbf{3 5}$, and cartoon representation of the construction of the supramolecular polymeric network via triply orthogonal self-assembly. (B) Fluorescence emission spectra of $\mathbf{3 7}$ and $\mathbf{3 9}$ irradiated at $441 \mathrm{~nm}$ and fluorescence emission spectra of $\mathbf{3 8}$ in the solid state, $\mathbf{4 0}$ in the solid state, and $\mathbf{4 0}$ in a gel, irradiated at $343 \mathrm{~nm}$. Adapted with permission from Zhou et al. (2016); copyright 2016, American Chemical Society. 
supramolecular polymers. In a similar manner to natural self-assembly processes, SCCs with superior properties have been efficiently developed by coordination-driven self-assembly, wherein the spontaneous connection between metal acceptors and organic donors via metal-ligand bonds resulted in the formation of non-covalent interactions and complicated structures (Smulders et al., 2013; Wei et al., 2015; Li et al., 2019a; Sun et al., 2019). In addition, over the past few decades, Stang (Sepehrpour et al., 2019), Fujita (Sawada et al., 2014), Raymond (Zhao et al., 2013), Mirkin (Mendez-Arroyo et al., 2014), Newkome (Chakraborty et al., 2017), Nitschke (Mosquera et al., 2016), and Yang (Chen L. -J. et al., 2015) have made enormous contributions to the development of a series of discrete SCCs with various shapes covering two-dimensional metallacycles and three-dimensional metallacages (Figure 11) (Cook et al., 2013). It is necessary to emphasize the difference between the metallic linkage applied in the cases of Figures 1, 2, 8 and that of SCCs. The former could comprise infinite linear polymers or cross-linked networks in which the metal centers and organic ligands bridged through metal-ligand coordination bonds, such as terpyridine-based metal-ligand interactions. The latter could form discrete systems where organometallic receptors and organic donors with specific angularity undergo self-assembly to generate finite supramolecular complexes. Due to the range of supramolecular structures, the stimulus-responsive nature of metal-ligand coordination interactions, and the introduction of metal atoms, SCCs have profound implications for the future development of light-emitting materials (Yan et al., 2015), sensors (Zhang et al., 2017b), molecular flasks (Inokuma et al., 2011), cell imaging (Zhang M. et al., 2016), and bioengineering (Zhou et al., 2019).

In addition, since the formation of metal-ligand coordination interactions does not interfere with other non-covalent interactions, complicated supramolecular polymer systems could be constructed using metal-ligand coordination-based interactions to construct metallacycles or metallacages as repetitive units with other non-covalent interactions, such as crown ether-based recognition systems (Wei et al., 2015). Utilizing two or more non-covalent bond forces to construct supramolecular polymers could not only give them richer stimulus responsiveness and other functions but could also provide a new flexible method for developing the topological structures of supramolecular polymers.

\section{Metallacycles as Building Blocks}

Zhou et al. (2016) reported a crown ether-based supramolecular polymer network using three orthogonal interactions, including coordination-driven self-assembly, hydrogen bonding, and hostguest interactions (Figure 12A). More specifically, a platinum acceptor 34 bearing a B21C7 moiety and a corresponding dipyridyl donor 35 with a pendant 2-ureido-4-pyrimidinone (UPy) unit was used to assemble a metallahexagon that was then converted into supramolecular polymer network 36 by the complementary hydrogen-bonding interactions of the UPy units. In addition, the resultant supramolecular polymer network 36 could be functionally modified using the free B21C7 moieties. Driven by host-guest interactions based on the recognition between the B21C7 moieties and dialkylammonium salts, two fluorescent supramolecular polymer networks (39 and 40) were developed by the introduction of perylene-decorated (37) and TPE-decorated (38) dialkylammonium salts, respectively. As evidenced by the fluorescence emission spectra of 37-40 (Figure 12B), the functional supramolecular polymer networks inherited the emission properties of independent precursors in the premise that the preassembly was not disrupted, suggesting that the construction strategy developed here was a feasible, efficient, and imitable methodology for fabricating fluorescent supramolecular polymers through tailored fluorophores.

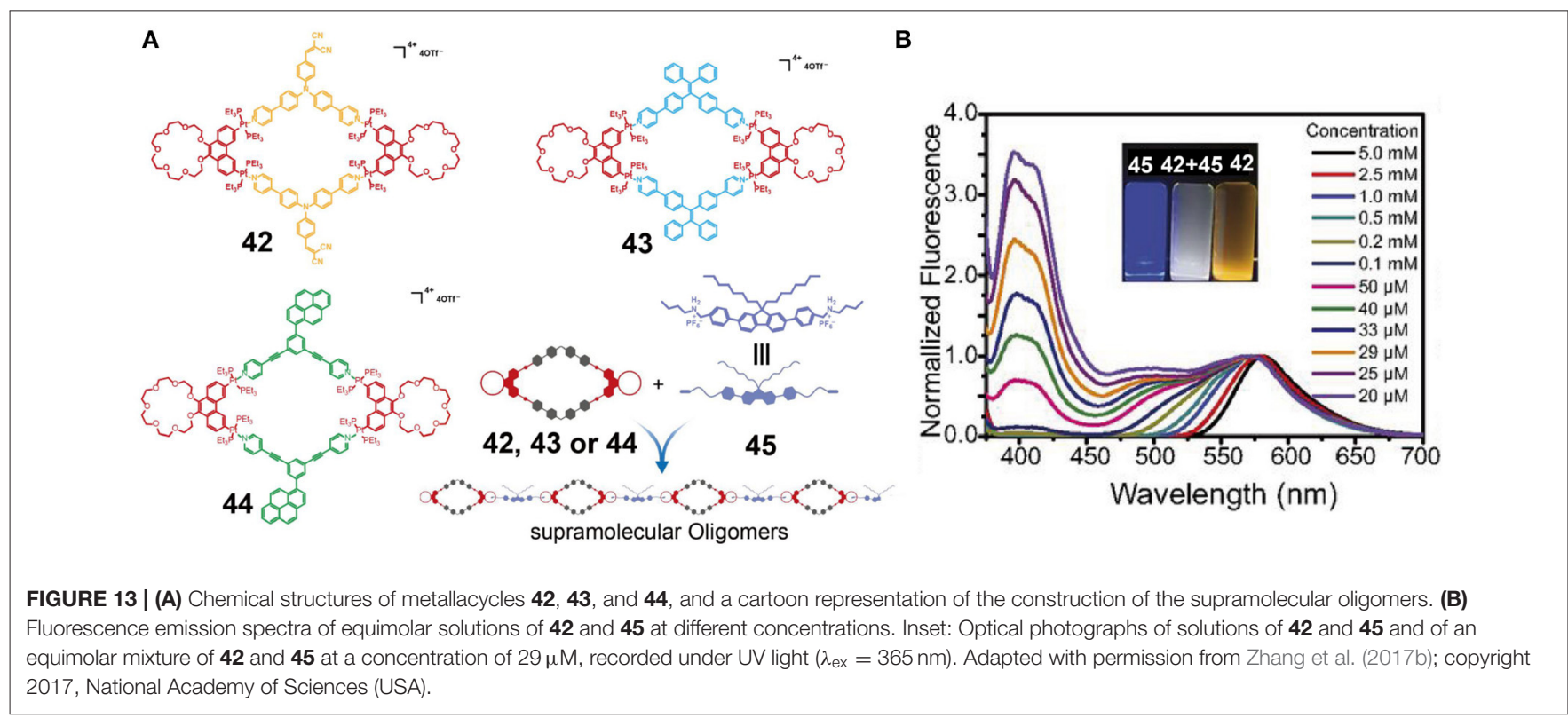


Zhang et al. (2017b) constructed metallacycles $\mathbf{4 2 - 4 4}$ by the coordination-driven self-assembly of well-established $120^{\circ}$ dipyridyl donors with phenanthrene-21-crown-7 (P21C7)based $60^{\circ}$ diplatinum(II) acceptors. Metallacycles 42-44 exhibited orange, cyan, and green emission colors, respectively, originating from the triphenylamine, tetraphenylethene, and pyrene fluorogens. Further polymerization could be efficiently achieved via crown ether-diaklyammonium salt-based host-guest interactions between the P21C7 units of metallacycles 42-44 and a fluorene-functionalized bis-ammonium salt 45 (Figure 13A). The supramolecular assemblies constructed using 42 and 45 mainly gave blue fluorescence in the dilute solution state (molar concentration of 42 or $45<25 \mu \mathrm{M}$ ), while they displayed orange emission at high concentrations (molar concentrations of $\mathbf{4 2}$ or $45>0.5 \mathrm{mM}$ ) since the ACQ properties of fluorene and the AIE properties of the triphenylamine moiety exhibited strong emissions only in dilute and concentrated solutions, respectively. As a result, this supramolecular system showed a strongly concentration-dependent emission from blue to orange. In addition, at a concentration of $29 \mu \mathrm{M}$, this supramolecular system simultaneously displayed white emission because its fluorescence emission covered the entire region from 400 to $700 \mathrm{~nm}$ (Figure 13B). Thus, the emission properties of given assemblies can be precise and can also be controlled on a large scale simply by adjusting the system concentration. However, the supramolecular assemblies formed from 43 to 45 and from 44 to 45 did not exhibit a pronounced tuneable emission over a large range due to their similar fluorescence color. Overall, the emissions of such supramolecular systems could be adjusted over a large range by changing the concentration of the system when complementary host-guest interactions (crown-ethers and diammonium salts), complementary emissions (blue and orange), and complementary fluorescence properties (AIE and ACQ) are combined into the same system, thereby providing a simple and effective method for the construction of fluorescent supramolecular assemblies. Such systems therefore play an important role in promoting the application of fluorescent supramolecular assemblies in the field of biotechnology and optoelectronics.

Light-emitting supramolecular assemblies play an important role in the fields of chemical sensors, biological imaging, and organic photoelectric materials. However, control of the fluorescence properties of supramolecular assemblies in a simple way and over a large range is one of the greatest challenges associated with the construction of fluorescent supramolecular assemblies. As an example, Xu et al. (2018b) first synthesized a series of P21C7-functionalized rhomboidal metallacycles $48 \mathrm{a}-48 \mathrm{~h}$ by variation of the substituents present on dipyridyl donor 46 and on P21C7-based $60^{\circ}$ diplatinum(II) acceptor 47 (Figure 14A). As a result, the structures of metallacycles 48 differed in the number and position of amino groups on the pyridine ligands or in the electronic effect and the conjugated structure of the aniline moiety at the para-position. Metallacycles bearing an endohedral amino group, with strong electrondonating substituents para to the aniline group, or presenting a longer conjugated structure, were found to exhibit higher quantum yields both in the solution and thin film states. Fluorescent supramolecular polymers emitting over a wide range from blue to red were then constructed through host-guest interactions between metallacycles $\mathbf{4 8}$ and bis-ammonium salts 49. These supramolecular polymers exhibited higher quantum yields than their corresponding discrete metallacycles both in

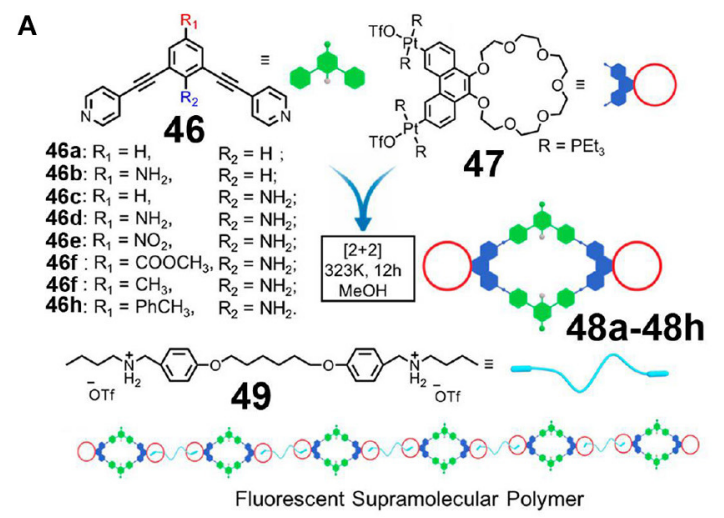

B

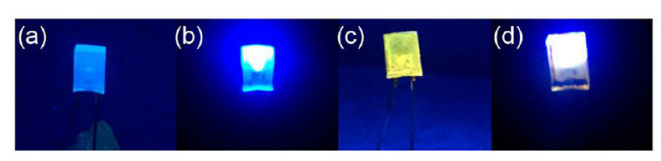

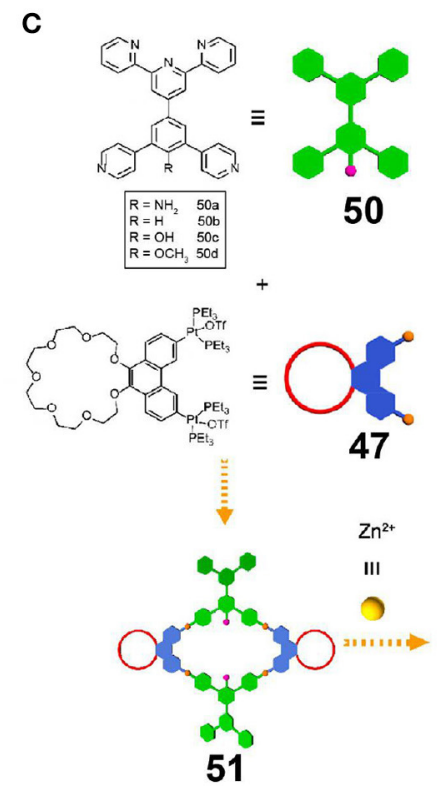
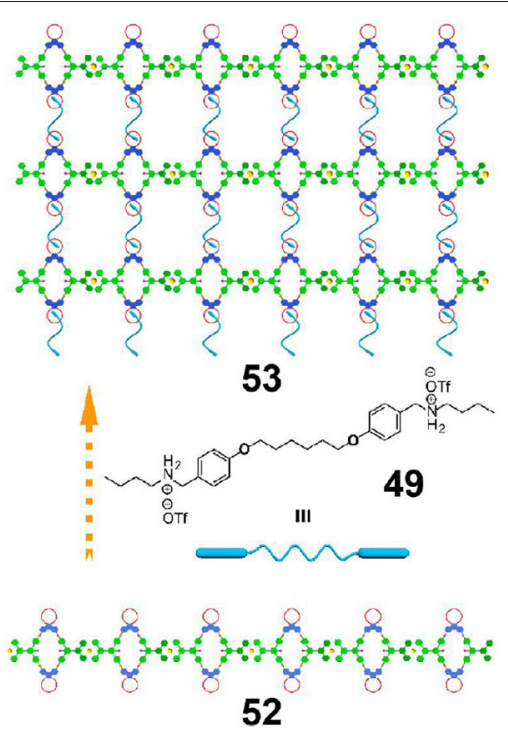

FIGURE 14 | (A) Cartoon representations of the construction of supramolecular metallacycles $\mathbf{4 8 a - 4 8 h}$ and the fluorescent supramolecular polymer. (B) Optical photographs of the unpainted $(a, b)$ and painted (c,d) UV-LED recorded under UV light $\left(\lambda_{\text {ex }}=365 \mathrm{~nm}\right)$ : (a,c) LED off, (b,d) LED on. Adapted with permission from Xu et al. (2018b); copyright 2018, American Chemical Society. (C) Cartoon representation of the construction of supramolecular metallacycles, linear supramolecular polymers, and cross-linked supramolecular polymeric networks. Adapted with permission from Zhang et al. (2019b); copyright 2019, American Chemical Society. 
the solution and thin film states, likely due to the formation of supramolecular polymers inhibiting tight packing of the assemblies, in turn facilitating an alternative stacking pattern that enhanced the emission efficiency. In addition, a white emission light-emitting diode (LED) was obtained by the incorporation of a yellow-emitting supramolecular polymer $48 \mathrm{~h}$ with good solubility and a high quantum yield along with a blue-emitting LED source (Figure 14B). The presented system therefore represents a new strategy for the preparation of tuneable fluorescent supramolecular polymers through modification of the functional groups of organic ligands and lays a foundation for the development of self-assembled polymer materials for photoelectric materials, biological imaging, biosensors, and other applications.

The bottom-up method of hierarchical self-assembly based on miscellaneous non-covalent interactions to prepare complex supramolecular structures is a powerful means of building novel functional supramolecular materials. However, the preparation of organometallic materials with precise structural control via hierarchical self-assembly remains a challenge, in particular in the case of systems containing heterometals. For example, Zhang et al. (2019b) reported the construction of four rhomboidal metallacycles $50 \mathrm{a}-\mathbf{5 0 \mathrm { d }}$ bearing a $\mathrm{P} 21 \mathrm{C} 7$ group and a conjugately-linked tripyridine moiety by platinum(II)-ligand coordination-driven self-assembly (Figure 14C). A linear bimetallic supramolecular polymer was then formed through metal-coordination between $\mathrm{Zn}$ and the tripyridine moiety. Finally, a bimetallic cross-linked supramolecular polymer was constructed through host-guest interactions between crown-ethers and diammonium salts. These three kinds of orthogonal non-covalent interactions do not interfere with one another, and so at high concentrations, the cross-linked supramolecular polymer can form a gel with multiple-stimulus-responsive properties in addition to good self-healing properties. Furthermore, the emission properties of such supramolecular polymers can be effectively controlled by changing the electron-donor ability of the pyridine ligands present in the metallacycles, thereby providing a new route to novel functional supramolecular polymers through various metal-ligand coordination interactions.

\section{Metallacages as Building Blocks}

$\mathrm{Lu}$ et al. (2018) reported a tetragonal prismatic metallacage, 57, bearing pendant $\mathrm{B} 21 \mathrm{C} 7$ moieties through the incorporation of cis-Pt $\left(\mathrm{PEt}_{3}\right)_{2}(\mathrm{OTf})_{2}$, TPE-functionalized sodium benzoate

A

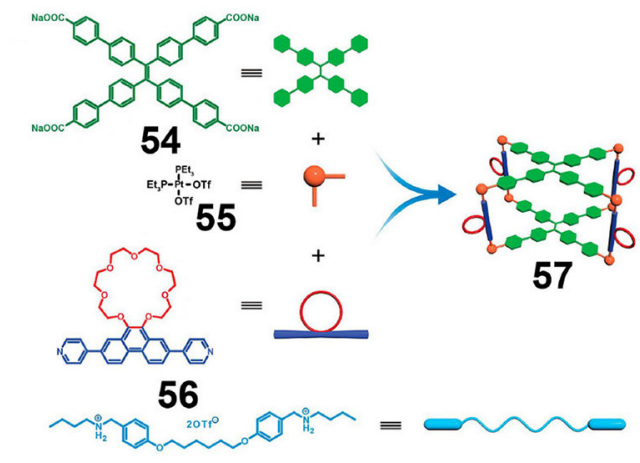

B

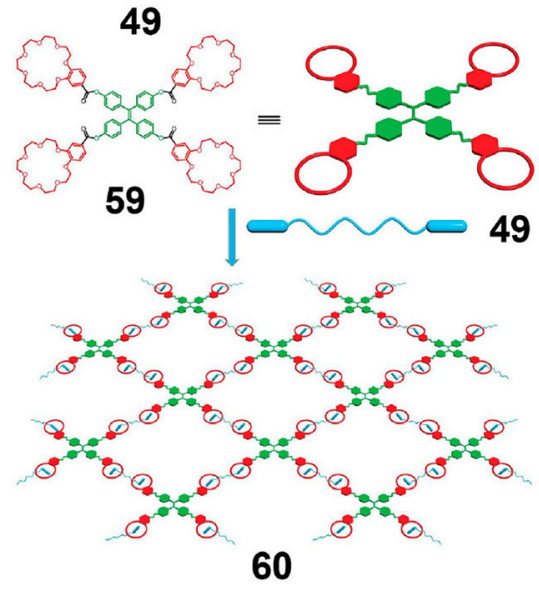

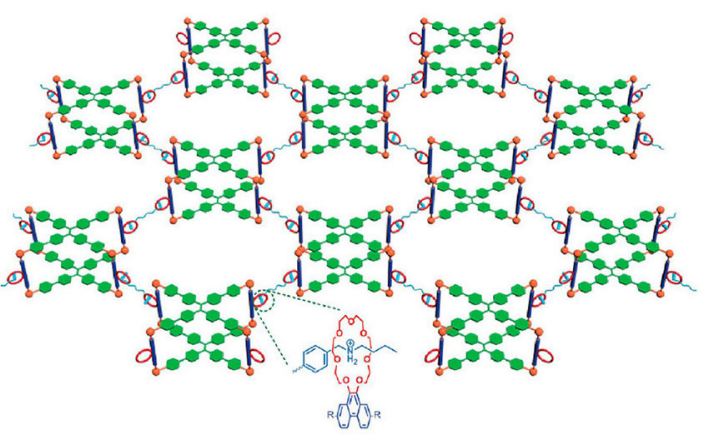

C

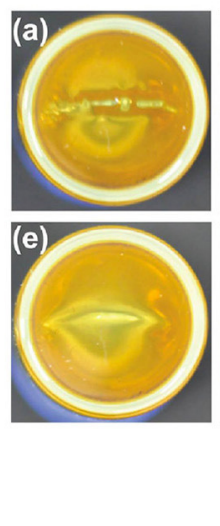

58
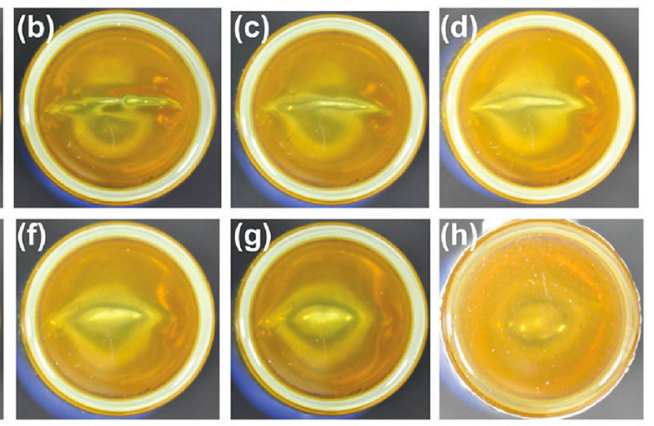

FIGURE 15 | (A) Cartoon representations of the construction of supramolecular metallacage $\mathbf{5 7}$ and metallacage-cored fluorescent supramolecular polymer $\mathbf{5 8}$. (B) Cartoon representation of the construction of cross-linked supramolecular polymer $\mathbf{6 0}$. (C) Optical photographs of the self-healing process of gel $\mathbf{5 8}$, and images showing the gel after cutting and allowing to stand for (a) 0, (b) 0.5, (c) 1.0, (d) 1.5, (e) 2, (f) 2.5, (g) 3, and (h) 4 min. Adapted with permission from Lu et al. (2018); copyright 2018, American Chemical Society. 
ligands, and linear dipyridyl ligands via metal-coordinationdriven self-assembly. Upon the addition of bis-ammonium linkers, a supramolecular polymer network (SPN) was formed via host-guest interactions (Figure 15A), and upon increasing the concentration of the SPN to a relatively high level, a supramolecular polymer gel was obtained that inherited the AIE properties originating from the TPE moieties. Moreover, due to the reversibility and environmental responsiveness of platinum(II)-ligand coordination interactions and the host-guest interactions based on the $\mathrm{B} 21 \mathrm{C} 7$ units and bis-ammonium salts, transitions between the metallacage-cored fluorescent supramolecular polymer gel and the disassembled sol with weak fluorescence were achieved by the addition of competing coordination compounds and thermal stimuli. Dynamic and reversible non-covalent interactions endowed the resultant gel with self-healing properties that allowed crack reparation over a short time (Figure 15C). To gain some insight into the effect of metallacages as cores in the gel, a model gel 60 (Figure 15B) was prepared via host-guest interactions between crown etherfunctionalized TPE derivatives and bis-ammonium linkers. In comparison with gel $\mathbf{6 0}$, gel $\mathbf{5 8}$ displayed a significantly stiffer structure due to the presence of rigid metallacages and higher branch functionalities. Thus, the above system provided a simple yet highly efficient strategy for obtaining multi-functional fluorescent supramolecular gels and laid a foundation for the development of dynamic but robust supramolecular materials. The authors also reported an improvement in the mechanical properties and self-healing properties of these fluorescent supramolecular gel systems when rigid metallacages were introduced through host-guest interactions, thereby showing promise for the application of metal-organic metallacages in intelligent soft materials, luminescent materials, drug carriers, and other fields.

\section{CONCLUSIONS}

We herein summarized the current published research works in the field of fluorescent supramolecular polymers formed by crown ether-based host-guest interactions. Depending on the building blocks employed, the fabrication strategies could be divided into three categories, including linking covalently bonded low-molecular weight compounds with the association units of hosts and guests, modifying hosts and guests with polymers, and the use of supramolecular coordination complexes (SCCs) bearing free crown ether units. The examples covered in this review comprehensively describe the superior performances of fluorescent materials based on the marriage of fluorophores and crown ether-based supramolecular macrocyclic compounds, which have demonstrated value in applications such as solidstate or gel-state fluorescent materials, fluorescent sensors, drug delivery monitoring, and optoelectronic devices. Aggregationinduced emission luminogens and conjugated oligomers such as tetraphenylethylene, triphenylamine, and oligomers of fluorene are usually selected to serve as the luminescent groups due to their facile modification and good luminescence. In addition, novel multifunctional supramolecular polymeric materials can be obtained through the combination of crownether-based host-guest interactions and other interactions, such as metal-ligand coordination interactions, and multiple hydrogen-bonding interactions. Furthermore, responsiveness to multiple stimuli is a particularly pronounced characteristic of crown ether-based supramolecular polymers, which arises from the dynamic and reversible non-covalent bonding present in such structures. Moreover, crown ether-based fluorescent supramolecular polymers bearing more topological structures possess the desired properties of both rigidity and reversibility, thereby rendering it possible for them to be easily functionalized and developed.

Although there have been important advances in fluorescent supramolecular polymers formed through crown etherbased host-guest interactions, efforts still should be devoted to exploiting uncharted terrains and addressing remaining challenges to ensure more extensive practical applications. Firstly, additional attention should be paid to utilizing existing fluorophores but also to creating novel fluorophores with unique characteristics. Secondly, there is a lack of depth and system in the research into fluorescent supramolecular polymers with various morphologies. However, the construction of amphipathic fluorescent nanoparticles from supramolecular polymers via crown ether-based recognition is one of the developments that could expand the potential applications in biology, such as cell imaging. Moreover, a feasible method for enriching the properties of fluorescent supramolecular polymeric materials is the introduction of more non-covalent interactions, such as $\pi-\pi$ stacking interactions, electrostatic interactions, and van der Waals forces. Finally, SCCs and, in particular, metallacages have been proven to be a novel kind of building block that is conducive to the emergence of new fluorescent supramolecular polymers. It can be anticipated that the combination of various fluorescent properties and the unique responsiveness and reversibility of crown ether-based supramolecular macrocyclic chemistry may broaden the application range of developing smart materials in areas such as photocatalysis, information transition, and data storage. We therefore expect continual endeavors to be carried out to ensure the development of novel fluorescent supramolecular polymers that exhibit desirable and enhanced properties as well as to ensure their subsequent practical application.

\section{AUTHOR CONTRIBUTIONS}

HQ, TH, YL, and SY designed the proposal and revised the manuscript. JZ wrote the first draft of the manuscript and prepared the figures. All authors read and approved the final manuscript prior to submission.

\section{ACKNOWLEDGMENTS}

We thank the National Natural Science Foundation of China (21971049 and 51903070) and the Zhejiang Provincial Natural Science Foundation of China (LQ18B040001 and LY16B040006) for financial support. 


\section{REFERENCES}

Bai, W., Wang, Z., Tong, J., Mei, J., Qin, A., Sun, J. Z., et al. (2015). A self-assembly induced emission system constructed by the host-guest interaction of AIE-active building blocks. Chem. Commun. 51, 1089-1091. doi: 10.1039/C4CC06510G

Chakraborty, S., Hong, W., Endres, K. J., Xie, T.-Z., Wojtas, L., Moorefield, C. N., et al. (2017). Terpyridine-based, flexible tripods: from a highly symmetric nanosphere to temperature-dependent, irreversible, 3D isomeric macromolecular nanocages. J. Am. Chem. Soc. 139, 3012-3020. doi: $10.1021 /$ jacs.6b11784

Chen, D., Zhan, J., Zhang, M., Zhang, J., Tao, J., Tang, D., et al. (2015). A fluorescent supramolecular polymer with aggregation induced emission (AIE) properties formed by crown ether-based host-guest interactions. Polym. Chem. 6, 25-29. doi: 10.1039/C4PY01206B

Chen, L.-J., Ren, Y.-Y., Wu, N.-W., Sun, B., Ma, J.-Q., Zhang, L., et al. (2015). Hierarchical self-assembly of discrete organoplatinum(II) metallacycles with polysaccharide via electrostatic interactions and their application for heparin detection. J. Am. Chem. Soc. 137, 11725-11735. doi: 10.1021/jacs.5b06565

Chen, P.-Z., Weng, Y.-X., Niu, L.-Y., Chen, Y.-Z., Wu, L.-Z., Tung, C.-H., et al. (2016). Light-harvesting systems based on organic nanocrystals to mimic chlorosomes. Angew. Chem. 128, 2809-2813. doi: 10.1002/ange.201510503

Cook, T. R., Zheng, Y.-R., and Stang, P. J. (2013). Metal-organic frameworks and self-assembled supramolecular coordination complexes: comparing and contrasting the design, synthesis, and functionality of metal-organic materials. Chem. Rev. 113, 734-777. doi: 10.1021/cr3002824

Deng, Y., Zhang, Q., Feringa, B. L., Tian, H., and Qu, D.-H. (2020). Toughening a self-healable supramolecular polymer by ionic clusterenhanced iron-carboxylate complexes. Angew. Chem. Int. Ed. 59, 5278-5283. doi: $10.1002 /$ anie. 201913893

Ding, Y., Wang, P., Tian, Y.-K., Tian, Y.-J., and Wang, F. (2013). Formation of stimuli-responsive supramolecular polymeric assemblies via orthogonal metal-ligand and host-guest interactions. Chem. Commun. 49, 5951-5953. doi: $10.1039 / \mathrm{c} 3 \mathrm{cc} 42511 \mathrm{~h}$

Dong, R., Ravinathan, S. P., Xue, L., Li, N., Zhang, Y., Zhou, L., et al. (2016). Dual-responsive aggregation-induced emission-active supramolecular nanoparticles for gene delivery and bioimaging. Chem. Commun. 52, 7950-7953. doi: 10.1039/C6CC02794F

Dong, S., Zheng, B., Xu, D., Yan, X., Zhang, M., and Huang, F. (2012). A crown ether appended super gelator with multiple stimulus responsiveness. $A d v$. Mater. 24, 3191-3195. doi: 10.1002/adma.201200837

Dsouza, R. N., Pischel, U., and Nau, W. M. (2011). Fluorescent dyes and their supramolecular host/guest complexes with macrocycles in aqueous solution. Chem. Rev. 111, 7941-7980. doi: 10.1021/cr200213s

Fermi, A., Bergamini, G., Roy, M., Gingras, M., and Ceroni, P. (2014). Turn-on phosphorescence by metal coordination to a multivalent terpyridine ligand: a new paradigm for luminescent sensors. J. Am. Chem. Soc. 136, 6395-6400. doi: $10.1021 /$ ja $501458 \mathrm{~s}$

Fu, H.-G., Chen, Y., and Liu, Y. (2019). Multistimuli-responsive and photocontrolled supramolecular luminescent gels constructed by anthracenebridged bis(dibenzo-24-crown-8) with secondary ammonium salt polymer. ACS Appl. Mater. Interfaces 11, 16117-16122. doi: 10.1021/acsami.9b04323

Gibson, H. W., Yamaguchi, N., Hamilton, L., and Jones, J. W. (2002). Cooperative self-assembly of dendrimers via pseudorotaxane formation from a homotritopic guest molecule and complementary monotopic host dendrons. J. Am. Chem. Soc. 124, 4653-4665. doi: 10.1021/ja012155s

Gibson, H. W., Yamaguchi, N., and Jones, J. W. (2003). Supramolecular pseudorotaxane polymers from complementary pairs of homoditopic molecules. J. Am. Chem. Soc. 125, 3522-3533. doi: 10.1021/ja 020900a

He, L., Li, L., Liu, X., Wang, J., Huang, H., and Bu, W. (2016). Acid-base-controlled and dibenzylammonium-assisted aggregation induced emission enhancement of poly(tetraphenylethene) with an impressive blue shift. Polym. Chem. 7, 3722-3730. doi: 10.1039/C6PY00275G

He, L., Liang, J., Cong, Y., Chen, X., and Bu, W. (2014). Concentration and acidbase controllable fluorescence of a metallosupramolecular polymer. Chem. Commun. 50, 10841-10844. doi: 10.1039/C4CC04243C
Huang, D., Zhang, Q., Deng, Y., Luo, Z., Li, B., Shen, X., et al. (2018). Polymeric crown ethers: LCST behavior in water and stimuli-responsiveness. Polym. Chem. 9, 2574-2579. doi: 10.1039/C8PY00412A

Huang, F., and Gibson, H. W. (2004). Formation of a supramolecular hyperbranched polymer from self-organization of an $\mathrm{AB} 2$ monomer containing a crown ether and two paraquat Moieties. J. Am. Chem. Soc. 126, 14738-14739. doi: $10.1021 / \mathrm{ja} 044830 \mathrm{e}$

Huang, F., Nagvekar, D. S., Zhou, X., and Gibson, H. W. (2007). Formation of a linear supramolecular polymer by self-assembly of two homoditopic monomers based on the bis(m-phenylene)-32-crown-10/paraquat recognition motif. Macromolecules 40, 3561-3567. doi: 10.1021/ma062080i

Inokuma, Y., Kawano, M., and Fujita, M. (2011). Crystalline molecular flasks. Nat. Chem. 3, 349-358. doi: 10.1038/nchem.1031

Ji, X., Dong, S., Wei, P., Xia, D., and Huang, F. (2013a). A novel diblock copolymer with a supramolecular polymer block and a traditional polymer block: preparation, controllable self-assembly in water, and application in controlled release. Adv. Mater. 25, 5725-5729. doi: 10.1002/adma.201301654

Ji, X., Li, Y., Wang, H., Zhao, R., Tang, G., and Huang, F. (2015). Facile construction of fluorescent polymeric aggregates with various morphologies by self-assembly of supramolecular amphiphilic graft copolymers. Polym. Chem. 6, 5021-5025. doi: 10.1039/C5PY00801H

Ji, X., Yao, Y., Li, J., Yan, X., and Huang, F. (2013b). A supramolecular cross-linked conjugated polymer network for multiple fluorescent sensing. J. Am. Chem. Soc. 135, 74-77. doi: 10.1021/ja3108559

Ji, X. F., Wang, P., Wang, H., and Huang, F.-H. (2015). A fluorescent supramolecular crosslinked polymer gel formed by crown ether based hostguest interactions and aggregation induced emission. Chin. J. Polym. Sci. 33, 890-898. doi: 10.1007/s10118-015-1639-6

Laurent, J., Blin, G., Chatelain, F., Vanneaux, V., Fuchs, A., Larghero, J., et al. (2017). Convergence of microengineering and cellular self-organization towards functional tissue manufacturing. Nat. Biomed. Eng. 1, 939-956. doi: 10.1038/s41551-017-0166-x

Li, B., He, T., Fan, Y., Yuan, X., Qiu, H., and Yin, S. (2019a). Recent developments in the construction of metallacycle/metallacage-cored supramolecular polymers via hierarchical self-assembly. Chem. Commun. 55, 8036-8059. doi: 10.1039/C9CC02472G

Li, B., He, T., Shen, X., Tang, D., and Yin, S. (2019b). Fluorescent supramolecular polymers with aggregation induced emission properties. Polym. Chem. 10, 796-818. doi: 10.1039/C8PY01396A

Li, B., Lin, C., Lu, C., Zhang, J., He, T., Qiu, H., et al. (2020). A rapid and reversible thermochromic supramolecular polymer hydrogel and its application in protected quick response codes. Mater. Chem. Front. 4, 869-874. doi: 10.1039/C9QM00699K

Li, J., Shi, K., Drechsler, M., Tang, B. Z., Huang, J., and Yan, Y. (2016). A supramolecular fluorescent vesicle based on a coordinating aggregation induced emission amphiphile: insight into the role of electrical charge in cancer cell division. Chem. Commun. 52, 12466-12469. doi: 10.1039/C6CC06432A

Li, X., Wang, L., Deng, Y., Luo, Z., Zhang, Q., Dong, S., et al. (2018). Preparation of cross-linked supramolecular polymers based on benzo-21-crown-7/secondary ammonium salt host-guest interactions. Chem. Commun. 54, 12459-12462. doi: 10.1039/C8CC07657J

Li, Y., Dong, Y., Miao, X., Ren, Y., Zhang, B., Wang, P., et al. (2018). Shapecontrollable and fluorescent supramolecular organic frameworks through aqueous host-guest complexation. Angew. Chem. Int. Ed. 57, 729-733. doi: 10.1002/anie.201710553

Liu, Y., Zhang, Y., Zhu, H., Wang, H., Tian, W., and Shi, B. (2018). A supramolecular hyperbranched polymer with multi-responsiveness constructed by pillar[5]arene-based host-guest recognition and its application in the breath figure method. Mater. Chem. Front. 2, 1568-1573. doi: 10.1039/C8QM00220G

Lou, X.-Y., and Yang, Y.-W. (2018). Manipulating aggregation-induced emission with supramolecular macrocycles. Adv. Optical Mater. 6:1800668. doi: 10.1002/adom.201800668

Lu, C., Zhang, M., Tang, D., Yan, X., Zhang, Z., Zhou, Z., et al. (2018). Fluorescent metallacage-core supramolecular polymer gel formed by orthogonal metal coordination and host-guest interactions. J. Am. Chem. Soc. 140, 7674-7680. doi: $10.1021 /$ jacs. 8 b03781 
Luo, J., Xie, Z., Lam, J. W. Y., Cheng, L., Chen, H., Qiu, C., et al. (2001). Aggregation-induced emission of 1-methyl-1,2,3,4,5-pentaphenylsilole. Chem. Commun., 1740-1741. doi: 10.1039/b105159h

Ma, X., Xie, J., Tang, N., and Wu, J. (2016). AIE-caused luminescence of a thermally-responsive supramolecular organogel. New J. Chem. 40, 6584-6587. doi: 10.1039/C6NJ01211F

Ma, X., and Zhao, Y. (2015). Biomedical applications of supramolecular systems based on host-guest interactions. Chem. Rev. 115, 7794-7839. doi: $10.1021 / \mathrm{cr} 500392 \mathrm{w}$

Ma, Y., Marszalek, T., Yuan, Z., Stangenberg, R., Pisula, W., Chen, L., et al. (2015). A crown ether decorated dibenzocoronene tetracarboxdiimide chromophore: synthesis, sensing, and self-organization. Chem. Asian J. 10, 139-143. doi: 10.1002/asia.201403037

Mendez-Arroyo, J., Barroso-Flores, J., Lifschitz, A. M., Sarjeant, A. A., Stern, C. L., and Mirkin, C. A. (2014). A multi-state, allosterically-regulated molecular receptor with switchable selectivity. J. Am. Chem. Soc. 136, 10340-10348. doi: $10.1021 /$ ja $503506 a$

Mosquera, J., Ronson, T. K., and Nitschke, J. R. (2016). Subcomponent flexibility enables conversion between D4-symmetric $\mathrm{Cd}_{8}^{\mathrm{II}} \mathrm{L}_{8}$ and $\mathrm{T}$-symmetric $\mathrm{Cd}_{4}^{\mathrm{II}} \mathrm{L}_{4}$ assemblies. J. Am. Chem. Soc. 138, 1812-1815. doi: 10.1021/jacs.5b12955

Pedersen, C. J. (1967). Cyclic polyethers and their complexes with metal salts. J. Am. Chem. Soc. 89, 7017-7036. doi: 10.1021/ja01002a035

Peng, H.-Q., Zheng, X., Han, T., Kwok, R. T. K., Lam, J. W. Y., Huang, X., et al. (2017). Dramatic differences in aggregation-induced emission and supramolecular polymerizability of tetraphenylethene-based stereoisomers. J. Am. Chem. Soc. 139, 10150-10156. doi: 10.1021/jacs.7b05792

Qu, D.-H., Wang, Q.-C., Zhang, Q.-W., Ma, X., and Tian, H. (2015). Photoresponsive host-guest functional systems. Chem. Rev. 115, 7543-7588. doi: $10.1021 /$ cr5006342

Roy, B., Noguchi, T., Yoshihara, D., Yamamoto, T., Sakamoto, J., and Shinkai, S. (2016). Amplified fluorescence emission of bolaamphiphilic perylene-azacrown ether derivatives directed towards molecular recognition events. PCCP 18, 13239-13245. doi: 10.1039/С6СР01545J

Sawada, T., Hisada, H., and Fujita, M. (2014). Mutual induced fit in a synthetic host-guest system. J. Am. Chem. Soc. 136, 4449-4451. doi: 10.1021/ja500376x

Sepehrpour, H., Fu, W., Sun, Y., and Stang, P. J. (2019). Biomedically relevant selfassembled metallacycles and metallacages. J. Am. Chem. Soc. 141, 14005-14020. doi: 10.1021 jacs.9b06222

Shi, B., Jie, K., Zhou, Y., Zhou, J., Xia, D., and Huang, F. (2016). Nanoparticles with near-infrared emission enhanced by pillararene-based molecular recognition in water. J. Am. Chem. Soc. 138, 80-83. doi: 10.1021/jacs.5b11676

Shi, B., Zhou, Z., Vanderlinden, R. T., Tang, J.-H., Yu, G., Acharyya, K., et al. (2019). Spontaneous supramolecular polymerization driven by discrete platinum metallacycle-based host-guest complexation. J. Am. Chem. Soc. 141, 11837-11841. doi: 10.1021/jacs.9b06181

Shi, C.-Y., Zhang, Q., Yu, C.-Y., Rao, S.-J., Yang, S., Tian, H., et al. (2020). An ultrastrong and highly stretchable polyurethane elastomer enabled by a zipper-like ring-sliding effect. Adv. Mater. 32:2000345. doi: 10.1002/adma.20 2000345

Smulders, M. M. J., Riddell, I. A., Browne, C., and Nitschke, J. R. (2013). Building on architectural principles for three-dimensional metallosupramolecular construction. Chem. Soc. Rev. 42, 1728-1754. doi: 10.1039/C2CS35254K

Sun, Y., Chen, C., and Stang, P. J. (2019). Soft materials with diverse suprastructures via the self-assembly of metal-organic complexes. Acc. Chem. Res. 52, 802-817. doi: 10.1021/acs.accounts.8b00663

Wang, H., Ji, X., Li, Z., Zhu, C. N., Yang, X., Li, T., et al. (2017). Preparation of a white-light-emitting fluorescent supramolecular polymer gel with a single chromophore and use of the gel to fabricate a protected quick response code. Mater. Chem. Front. 1, 167-171. doi: 10.1039/C6QM00164E

Wang, L., Cheng, L., Li, G., Liu, K., Zhang, Z., Li, P., et al. (2020). A self-cross-linking supramolecular polymer network enabled by crownether-based molecular recognition. J. Am. Chem. Soc. 142, 2051-2058. doi: $10.1021 /$ jacs.9b12164

Wang, X., Hu, J., Liu, T., Zhang, G., and Liu, S. (2012). Highly sensitive and selective fluorometric off-on $\mathrm{K}+$ probe constructed via host-guest molecular recognition and aggregation-induced emission. J. Mater. Chem. 22, 8622-8628. doi: $10.1039 / \mathrm{c} 2 \mathrm{jm} 16510 \mathrm{~d}$
Wei, P., Yan, X., and Huang, F. (2015). Supramolecular polymers constructed by orthogonal self-assembly based on host-guest and metal-ligand interactions. Chem. Soc. Rev. 44, 815-832. doi: 10.1039/C4CS00327F

Xiao, T., Zhou, L., Sun, X.-Q., Huang, F., Lin, C., and Wang, L. (2020). Supramolecular polymers fabricated by orthogonal self-assembly based on multiple hydrogen bonding and macrocyclic host-guest interactions. Chin. Chem. Lett. 31, 1-9. doi: 10.1016/j.cclet.2019.05.011

Xu, L., Chen, D., Zhang, Q., He, T., Lu, C., Shen, X., et al. (2018a). A fluorescent cross-linked supramolecular network formed by orthogonal metalcoordination and host-guest interactions for multiple ratiometric sensing. Polym. Chem. 9, 399-403. doi: 10.1039/C7PY01788J

Xu, L., Shen, X., Zhou, Z., He, T., Zhang, J., Qiu, H., et al. (2018b). Metallacycle-cored supramolecular polymers: fluorescence tuning by variation of substituents. J. Am. Chem. Soc. 140, 16920-16924. doi: 10.1021/jacs.8b10842

Yamaguchi, N., and Gibson, H. W. (1999). Formation of supramolecular polymers from homoditopic molecules containing secondary ammonium ions and crown ether moieties. Angew. Chem. Int. Ed. 38, 143-147.

Yamaguchi, N., Nagvekar, D. S., and Gibson, H. W. (1998). Self-organization of a heteroditopic molecule to linear polymolecular arrays in solution. Angew. Chem. Int. Ed. 37, 2361-2364.

Yan, X., Cook, T. R., Pollock, J. B., Wei, P., Zhang, Y., Yu, Y., et al. (2014). Responsive supramolecular polymer metallogel constructed by orthogonal coordination-driven self-assembly and host/guest interactions. J. Am. Chem. Soc. 136, 4460-4463. doi: $10.1021 / \mathrm{ja} 412249 \mathrm{k}$

Yan, X., Cook, T. R., Wang, P., Huang, F., and Stang, P. J. (2015). Highly emissive platinum(II) metallacages. Nat. Chem. 7, 342-348. doi: 10.1038/nchem.2201

Yan, X., Li, S., Cook, T. R., Ji, X., Yao, Y., Pollock, J. B., et al. (2013). Hierarchical self-assembly: well-defined supramolecular nanostructures and metallohydrogels via amphiphilic discrete organoplatinum(II) metallacycles. J. Am. Chem. Soc. 135, 14036-14039. doi: 10.1021/ja406877b

Yan, X., Wang, F., Zheng, B., and Huang, F. (2012a). Stimuli-responsive supramolecular polymeric materials. Chem. Soc. Rev. 41, 6042-6065. doi: $10.1039 / \mathrm{c} 2 \mathrm{cs} 35091 \mathrm{~b}$

Yan, X., Xu, D., Chi, X., Chen, J., Dong, S., Ding, X., et al. (2012b). A multiresponsive, shape-persistent, and elastic supramolecular polymer network gel constructed by orthogonal self-assembly. Adv. Mater. 24, 362-369. doi: 10.1002/adma.201103220

Yu, G., Jie, K., and Huang, F. (2015). Supramolecular amphiphiles based on host-guest molecular recognition motifs. Chem. Rev. 115, 7240-7303. doi: $10.1021 / \mathrm{cr} 5005315$

Yu, G., Yan, X., Han, C., and Huang, F. (2013). Characterization of supramolecular gels. Chem. Soc. Rev. 42, 6697-6722. doi: 10.1039/c3cs60080g

Yu, G., Zhang, M., Saha, M. L., Mao, Z., Chen, J., Yao, Y., et al. (2017). Antitumor activity of a unique polymer that incorporates a fluorescent self-assembled metallacycle. J. Am. Chem. Soc. 139, 15940-15949. doi: 10.1021/jacs.7b09224

Yu, X., Chen, L., Zhang, M., and Yi, T. (2014). Low-molecular-mass gels responding to ultrasound and mechanical stress: towards self-healing materials. Chem. Soc. Rev. 43, 5346-5371. doi: 10.1039/C4CS00066H

Zhan, J., Li, Q., Hu, Q., Wu, Q., Li, C., Qiu, H., et al. (2014a). A stimuli-responsive orthogonal supramolecular polymer network formed by metal-ligand and host-guest interactions. Chem. Commun. 50, 722-724. doi: $10.1039 / \mathrm{C} 3 \mathrm{CC} 47468 \mathrm{~B}$

Zhan, J., Zhang, M., Zhou, M., Liu, B., Chen, D., Liu, Y., et al. (2014b). A multiple-responsive self-healing supramolecular polymer gel network based on multiple orthogonal interactions. Macromol. Rapid Commun. 35, 1424-1429. doi: 10.1002/marc. 201400216

Zhang, C., Li, S., Zhang, J., Zhu, K., Li, N., and Huang, F. (2007). Benzo-21-crown7/secondary dialkylammonium salt [2]pseudorotaxane- and [2] rotaxane-type threaded structures. Org. Lett. 9, 5553-5556. doi: 10.1021/ol702510c

Zhang, J., Zhang, K., Huang, X., Cai, W., Zhou, C., Liu, S., et al. (2012). Supramolecular light-emitting polymers for solution-processed optoelectronic devices. J. Mater. Chem. 22, 12759-12766. doi: 10.1039/c2jm31773g

Zhang, J., Zhu, J., Lu, C., Gu, Z., He, T., Yang, A., et al. (2016). A hyperbranched fluorescent supramolecular polymer with aggregation induced emission (AIE) properties. Polym. Chem. 7, 4317-4321. doi: 10.1039/C6PY00872K

Zhang, M., Li, S., Yan, X., Zhou, Z., Saha, M. L., Wang, Y.-C., et al. (2016). Fluorescent metallacycle-cored polymers via covalent linkage and their use 
as contrast agents for cell imaging. Proc. Natl. Acad. Sci. U.S.A. 113:11100. doi: $10.1073 /$ pnas. 1612898113

Zhang, M., Saha, M. L., Wang, M., Zhou, Z., Song, B., Lu, C., et al. (2017a). Multicomponent platinum(II) cages with tunable emission and amino acid sensing. J. Am. Chem. Soc. 139, 5067-5074. doi: 10.1021/jacs. $6 \mathrm{~b} 12536$

Zhang, M., Yin, S., Zhang, J., Zhou, Z., Saha, M. L., Lu, C., et al. (2017b). Metallacycle-cored supramolecular assemblies with tunable fluorescence including white-light emission. Proc. Natl. Acad. Sci. U.S.A. 114:3044. doi: $10.1073 /$ pnas.1702510114

Zhang, Q., Deng, Y.-X., Luo, H.-X., Shi, C.-Y., Geise, G. M., Feringa, B. L., et al. (2019a). Assembling a natural small molecule into a supramolecular network with high structural order and dynamic functions. J. Am. Chem. Soc. 141, 12804-12814. doi: 10.1021/jacs.9b05740

Zhang, Q., Shi, C.-Y., Qu, D.-H., Long, Y.-T., Feringa, B. L., and Tian, H. (2018). Exploring a naturally tailored small molecule for stretchable, self-healing, and adhesive supramolecular polymers. Sci. Adv. 4:eaat8192. doi: $10.1126 /$ sciadv.aat 8192

Zhang, Q., Tang, D., Zhang, J., Ni, R., Xu, L., He, T., et al. (2019b). SelfHealing heterometallic supramolecular polymers constructed by hierarchical assembly of triply orthogonal interactions with tunable photophysical properties. J. Am. Chem. Soc. 141, 17909-17917. doi: 10.1021/jacs. 9 b09671

Zhao, C., Sun, Q.-F., Hart-Cooper, W. M., DiPasquale, A. G., Toste, F. D., Bergman, R. G., et al. (2013). Chiral amide directed assembly of a diastereoand enantiopure supramolecular host and its application to enantioselective catalysis of neutral substrates. J. Am. Chem. Soc. 135, 18802-18805 doi: 10.1021/ja411631v

Zheng, B., Wang, F., Dong, S., and Huang, F. (2012). Supramolecular polymers constructed by crown ether-based molecular recognition. Chem. Soc. Rev. 41, 1621-1636. doi: 10.1039/C1CS15220C

Zhou, Z., Liu, J., Huang, J., Rees, T. W., Wang, Y., Wang, H., et al. (2019). A self-assembled Ru-Pt metallacage as a lysosome-targeting photosensitizer for 2-photon photodynamic therapy. Proc. Natl. Acad. Sci. U.S.A. 116:20296. doi: 10.1073/pnas.1912549116

Zhou, Z., Yan, X., Cook, T. R., Saha, M. L., and Stang, P. J. (2016). Engineering functionalization in a supramolecular polymer: hierarchical self-organization of triply orthogonal non-covalent interactions on a supramolecular coordination complex platform. J. Am. Chem. Soc. 138, 806-809. doi: 10.1021/jacs.5b12986

Conflict of Interest: The authors declare that the research was conducted in the absence of any commercial or financial relationships that could be construed as a potential conflict of interest.

Copyright (c) 2020 Zhang, Qiu, He, Li and Yin. This is an open-access article distributed under the terms of the Creative Commons Attribution License (CC BY). The use, distribution or reproduction in other forums is permitted, provided the original author(s) and the copyright owner(s) are credited and that the original publication in this journal is cited, in accordance with accepted academic practice. No use, distribution or reproduction is permitted which does not comply with these terms. 